KACHANOVSKY N.A. ${ }^{1}$, KACHANOVSKA T.O. ${ }^{2}$

\title{
INTERCONNECTION BETWEEN WICK MULTIPLICATION AND INTEGRATION ON SPACES OF NONREGULAR GENERALIZED FUNCTIONS IN THE LÉVY WHITE NOISE ANALYSIS
}

\begin{abstract}
We deal with spaces of nonregular generalized functions in the Lévy white noise analysis, which are constructed using Lytvynov's generalization of a chaotic representation property. Our aim is to describe a relationship between Wick multiplication and integration on these spaces. More exactly, we show that when employing the Wick multiplication, it is possible to take a time-independent multiplier out of the sign of an extended stochastic integral; establish an analog of this result for a Pettis integral (a weak integral); and prove a theorem about a representation of the extended stochastic integral via the Pettis integral from the Wick product of the original integrand by a Lévy white noise. As examples of an application of our results, we consider some stochastic equations with Wick type nonlinearities.
\end{abstract}

Key words and phrases: Lévy process, extended stochastic integral, Pettis integral, Wick product.

\footnotetext{
${ }^{1}$ Institute of Mathematics, National Academy of Sciences of Ukraine, 3 Tereschenkivska str., 01601, Kyiv, Ukraine

${ }^{2}$ Institute of Phylology, Taras Shevchenko National University, 14 Taras Shevchenko Blvd., Kyiv, 01601, Ukraine

E-mail: nkachano@gmail.com (Kachanovsky N.A.), tkatchanovska@gmail.com (Kachanovska T.O.)
}

\section{INTRODUCTION}

A theory of test and generalized functions depending on infinitely many variables (i.e., with arguments belonging to infinite-dimensional spaces) is highly sought in many areas of modern physics and mathematics. One of successful approaches to building of such a theory consists in introduction of spaces of the above-mentioned functions in such a way that the dual pairing between test and generalized functions is generated by integration with respect to some probability measure on a dual nuclear space. First it was the Gaussian measure, the corresponding theory is called the Gaussian white noise analysis (e.g., [2,16,26-28]), then it were realized numerous generalizations. In particular, important results can be obtained if one uses the Lévy white noise measure (e.g., $[6,7,29])$, the corresponding theory is called the Lévy white noise analysis.

A very important role in the Gaussian analysis belongs to a so-called chaotic representation property (CRP). This property consists, roughly speaking, in the following: any square integrable random variable can be decomposed in a series of repeated Itô's stochastic integrals from nonrandom functions (see, e.g., [30] for a detailed presentation). Using CRP, one can construct various spaces of test and generalized functions, introduce stochastic integrals and derivatives on these spaces, etc. In the Lévy analysis there is no CRP (more exactly, the only 
Lévy processes with CRP are Wiener and Poisson processes) [35]; but there are different generalizations of this property: Itô's generalization [18], Nualart-Schoutens' generalization [31,32], Lytvynov's generalization [29], Oksendal's generalization [6,7], etc. The interconnections between these generalizations are described in, e.g., [1, 6, 7, 21, 29, 34,36]. Now, depending on problems under consideration, one can select a most suitable generalization of CRP, construct corresponding spaces of test and generalized functions, and introduce necessary operators and operations on these spaces.

In the present paper we deal with one of the most useful and challenging generalizations of CRP in the Lévy white noise analysis, which is proposed by E. W. Lytvynov [29] (see also [5]). The idea of this generalization is to decompose random variables, square integrable with respect to the Lévy white noise measure, in series of special orthogonal functions, by analogy with decompositions of random variables, square integrable with respect to the Gaussian measure, by Hermite polynomials (remind that the last decompositions are equivalent to the decompositions by repeated Itô's stochastic integrals). Like using CRP in the Gaussian analysis, one can use Lytvynov's generalization of CRP in order to construct and study spaces of regular and nonregular test and generalized functions [19], various operators and operations on these spaces, etc. In particular, the extended stochastic integral and the Hida stochastic derivative on the spaces of regular test and generalized functions are introduced and studied in [10,19], operators of stochastic differentiation-in $[8,9,13]$, some elements of a Wick calculus and its relationship with operators of stochastic differentiation-in [11]. As for the spaces of nonregular test and generalized functions-the corresponding results are presented in [19,22-24].

As is well known, in the Gaussian white noise analysis, in the same way as in various versions of a non-Gaussian analysis, a natural multiplication on spaces of generalized functions is a so-called Wick multiplication. In particular, in many cases, using the Wick multiplication, one can take a time-independent multiplier out of the sign of an extended stochastic integral. Moreover, such a result holds true for a Pettis integral (a weak integral). Also, the extended stochastic integral can be presented as a Pettis integral (or a formal Pettis integral-depending on the concrete situation) from the Wick product of the original integrand by the derivative (in the sense of generalized functions) of the integrator. On the above-mentioned spaces of regular generalized functions in the Lévy analysis such results were obtained in [12]. The aim of the present paper is to transfer the results of [12] to the spaces of nonregular generalized functions, which are constructed using Lytvynov's generalization of CRP. In a sense, this paper is a continuation of the paper [22].

The paper is organized in the following manner. In the first section we introduce a Lévy process $L$ and construct a probability triplet connected with $L$, convenient for our considerations; then we describe Lytvynov's generalization of CRP; construct a nonregular rigging of the space of square integrable random variables (the positive and negative spaces of this rigging are the spaces of nonregular test and generalized functions respectively); describe the extended stochastic integral with respect to $L$ on the spaces of nonregular generalized functions; and recall necessary notions of the Wick calculus. In the second section we show that when employing the Wick multiplication, it is possible to take a time-independent multiplier out of the sign of the extended stochastic integral and of the Pettis integral; prove a theorem about a representation of the extended stochastic integral via the Pettis integral; and consider examples. 


\section{PRELIMINARIES}

In this paper we denote by $\|\cdot\|_{H}$ or $|\cdot|_{H}$ the norm in a space $H$; by $(\cdot, \cdot)_{H}$ the real, i.e., bilinear scalar product in a space $H$; and by $\langle\langle\cdot, \cdot\rangle\rangle_{H}$ the dual pairing generated by the scalar product in a space $H$. Further, we use a designation pr lim (resp., ind lim) for a projective (resp., inductive) limit of a family of spaces, this designation implies that the limit space is endowed with the projective (resp., inductive) limit topology (see, e.g., [3] for a detailed description).

\subsection{A Lévy process and its probability space}

Denote $\mathbb{R}_{+}:=[0,+\infty)$. Let $L=\left(L_{u}\right)_{u \in \mathbb{R}_{+}}$be a real-valued locally square integrable Lévy process (i.e., a continuous in probability random process on $\mathbb{R}_{+}$with stationary independent increments and such that $L_{0}=0$, see, e.g., [4] for details) without Gaussian part and drift. As is well known (e.g., [7]), the characteristic function of $L$ is

$$
\mathbb{E}\left[e^{i \theta L_{u}}\right]=\exp \left[u \int_{\mathbb{R}}\left(e^{i \theta x}-1-i \theta x\right) v(d x)\right],
$$

where $v$ is the Lévy measure of $L$, which is a measure on $(\mathbb{R}, \mathcal{B}(\mathbb{R}))$, here and below $\mathcal{B}$ denotes the Borel $\sigma$-algebra; $\mathbb{E}$ denotes the expectation. We assume that $v$ is a Radon measure whose support contains an infinite number of points, $v(\{0\})=0$, there exists $\varepsilon>0$ such that $\int_{\mathbb{R}} x^{2} e^{\varepsilon|x|} v(d x)<\infty$, and $\int_{\mathbb{R}} x^{2} v(d x)=1$.

Define a measure of the white noise of $L$. Let $\mathcal{D}$ denote the set of all real-valued infinitedifferentiable functions on $\mathbb{R}_{+}$with compact supports. As is well known, $\mathcal{D}$ can be endowed by the projective limit topology generated by a family of Sobolev spaces (e.g., [3]; see also Subsection 1.3). Let $\mathcal{D}^{\prime}$ be the set of linear continuous functionals on $\mathcal{D}$. For $\omega \in \mathcal{D}^{\prime}$ and $\varphi \in \mathcal{D}$ denote $\omega(\varphi)$ by $\langle\omega, \varphi\rangle$. It is worth noting that $\mathcal{D}$ and $\mathcal{D}^{\prime}$ are the positive and negative spaces of a chain

$$
\mathcal{D}^{\prime} \supset L^{2}\left(\mathbb{R}_{+}\right) \supset \mathcal{D},
$$

where $L^{2}\left(\mathbb{R}_{+}\right)$is the space of (classes of) real-valued functions on $\mathbb{R}_{+}$, square integrable with respect to the Lebesgue measure (e.g., [3]), and therefore $\langle\cdot, \cdot\rangle$ is the dual pairing generated by the scalar product in $L^{2}\left(\mathbb{R}_{+}\right)$. The notation $\langle\cdot, \cdot\rangle$ will be preserved for dual pairings in tensor powers of the complexification of chain (2).

Definition 1. A probability measure $\mu$ on $\left(\mathcal{D}^{\prime}, \mathcal{C}\left(\mathcal{D}^{\prime}\right)\right.$ ), where $\mathcal{C}$ denotes the cylindrical $\sigma$ algebra, with the Fourier transform

$$
\int_{\mathcal{D}^{\prime}} e^{i\langle\omega, \varphi\rangle} \mu(d \omega)=\exp \left[\int_{\mathbb{R}_{+} \times \mathbb{R}}\left(e^{i \varphi(u) x}-1-i \varphi(u) x\right) d u v(d x)\right], \quad \varphi \in \mathcal{D},
$$

is called the measure of a Lévy white noise.

The existence of $\mu$ follows from the Bochner-Minlos theorem (e.g., [17]), see [29]. Below we assume that the $\sigma$-algebra $\mathcal{C}\left(\mathcal{D}^{\prime}\right)$ is completed with respect to $\mu$.

Denote by $\left(L^{2}\right):=L^{2}\left(\mathcal{D}^{\prime}, \mathcal{C}\left(\mathcal{D}^{\prime}\right), \mu\right)$ the space of (classes of) complex-valued functions on $\mathcal{D}^{\prime}$, square integrable with respect to $\mu$ (in what follows, this notation will be used very often). Let $f \in L^{2}\left(\mathbb{R}_{+}\right)$and a sequence $\left(\varphi_{k} \in \mathcal{D}\right)_{k \in \mathbb{N}}$ converge to $f$ in $L^{2}\left(\mathbb{R}_{+}\right)$as $k \rightarrow \infty$ (remind that $\mathcal{D}$ is a dense set in $\left.L^{2}\left(\mathbb{R}_{+}\right)\right)$. One can show $[6,7,21,29]$ that $\langle 0, f\rangle:=\left(L^{2}\right)-\lim _{k \rightarrow \infty}\left\langle 0, \varphi_{k}\right\rangle$ is a well-defined element of $\left(L^{2}\right)$. 
Denote by $1_{A}$ the indicator of a set $A$, and put $1_{[0,0)} \equiv 0$. It follows from (1) and (3) that $\left(\left\langle 0,1_{[0, u)}\right\rangle\right)_{u \in \mathbb{R}_{+}}$can be identified with a Lévy process on the probability space (triplet) $\left(\mathcal{D}^{\prime}, \mathcal{C}\left(\mathcal{D}^{\prime}\right), \mu\right)$, see, e.g., [6,7]. So, for each $u \in \mathbb{R}_{+}$we have $L_{u}=\left\langle 0,1_{[0, u)}\right\rangle \in\left(L^{2}\right)$.

Note that the derivative in the sense of generalized functions of a Lévy process (a Lévy white noise) is $\dot{L} .(\omega)=\langle\omega, \delta.\rangle \equiv \omega(\cdot)$, where $\delta$ is the Dirac delta-function. Therefore $\dot{L}$ is a generalized random process (in the sense of [14]) with trajectories from $\mathcal{D}^{\prime}$, and $\mu$ is the measure of $\dot{L}$ in the classical sense of this notion [15].

Remark 1. A Lévy process $L$ without Gaussian part and drift is a Poisson process if its Lévy measure $v$ is a point mass at 1 . This measure does not satisfy the assumptions accepted above (its support does not contain an infinite number of points); nevertheless, all results of the present paper have natural analogs in the Poissonian analysis. The reader can find more information about peculiarities of the Poissonian case in [21], Subsection 1.2.

\subsection{Lytvynov's generalization of CRP}

Denote by $\widehat{\otimes}$ the symmetric tensor multiplication, by a subscript $\mathbb{C}$-complexifications of spaces. Set $\mathbb{Z}_{+}:=\mathbb{N} \cup\{0\}$. Denote by $\mathcal{P}$ the set of complex-valued polynomials on $\mathcal{D}^{\prime}$ that consists of zero and elements of the form

$$
f(\omega)=\sum_{n=0}^{N_{f}}\left\langle\omega^{\otimes n}, f^{(n)}\right\rangle, \quad \omega \in \mathcal{D}^{\prime}, f^{(n)} \in \mathcal{D}_{\mathbb{C}}^{\widehat{\otimes} n}, N_{f} \in \mathbb{Z}_{+}, f^{\left(N_{f}\right)} \neq 0,
$$

here $N_{f}$ is called the power of a polynomial $f ;\left\langle\omega^{\otimes 0}, f^{(0)}\right\rangle:=f^{(0)} \in \mathcal{D}_{\mathbb{C}}^{\widehat{\otimes} 0}:=\mathbb{C}$. The measure $\mu$ of a Lévy white noise has a holomorphic at zero Laplace transform (this follows from (3) and properties of the measure $v$, see also [29]), therefore $\mathcal{P}$ is a dense set in $\left(L^{2}\right)$ [33]. Denote by $\mathcal{P}_{n}, n \in \mathbb{Z}_{+}$, the set of polynomials of power smaller than or equal to $n$, by $\overline{\mathcal{P}}_{n}$ the closure of $\mathcal{P}_{n}$ in $\left(L^{2}\right)$. Let for $n \in \mathbb{N} \mathbf{P}_{n}:=\overline{\mathcal{P}}_{n} \ominus \overline{\mathcal{P}}_{n-1}$ (the orthogonal difference in $\left(L^{2}\right)$ ); put $\mathbf{P}_{0}:=\overline{\mathcal{P}}_{0}$. It is clear that

$$
\left(L^{2}\right)=\bigoplus_{n=0}^{\infty} \mathbf{P}_{n} .
$$

Let $f^{(n)} \in \mathcal{D}_{\mathbb{C}}^{\widehat{\otimes} n}, n \in \mathbb{Z}_{+}$. Denote by $:\left\langle 0^{\otimes n}, f^{(n)}\right\rangle:$ the orthogonal projection of a monomial $\left\langle 0^{\otimes n}, f^{(n)}\right\rangle$ onto $\mathbf{P}_{n}$. Let us define real (bilinear) scalar products $(\cdot, \cdot)_{\text {ext }}$ on $\mathcal{D}_{\mathbb{C}}^{\widehat{\otimes} n}, n \in \mathbb{Z}_{+}$, by setting for $f^{(n)}, g^{(n)} \in \mathcal{D}_{\mathbb{C}}^{\widehat{\otimes} n}$

$$
\left(f^{(n)}, g^{(n)}\right)_{\text {ext }}:=\frac{1}{n !} \int_{\mathcal{D}^{\prime}}:\left\langle\omega^{\otimes n}, f^{(n)}\right\rangle::\left\langle\omega^{\otimes n}, g^{(n)}\right\rangle: \mu(d \omega) .
$$

The proof of the well-posedness of this definition coincides up to obvious modifications with the proof of the corresponding statement in [29].

Denote by $|\cdot|_{\text {ext }}$ the norms corresponding to scalar products (5), i.e., $|\cdot|_{\text {ext }}:=\sqrt{\left(\cdot, \overline{C_{e x t}}\right.}$. Let $\mathcal{H}_{\text {ext }}^{(n)}, n \in \mathbb{Z}_{+}$, be the completions of $\mathcal{D}_{\mathrm{C}}^{\widehat{\otimes} n}$ with respect to these norms. For $F^{(n)} \in \mathcal{H}_{\text {ext }}^{(n)}$ define a Wick monomial $:\left\langle 0^{\otimes n}, F^{(n)}\right\rangle: \stackrel{\text { def }}{=}\left(L^{2}\right)-\lim _{k \rightarrow \infty}:\left\langle 0^{\otimes n}, f_{k}^{(n)}\right\rangle:$, where $\mathcal{D}_{\mathbb{C}}^{\widehat{\otimes} n} \ni f_{k}^{(n)} \rightarrow F^{(n)}$ as $k \rightarrow \infty$ in $\mathcal{H}_{\text {ext }}^{(n)}$. The well-posedness of this definition can be proved by the method of "mixed sequences". It is easy to show that $:\left\langle 0^{\otimes 0}, F^{(0)}\right\rangle:=\left\langle 0^{\otimes 0}, F^{(0)}\right\rangle=F^{(0)}$ and $:\left\langle\circ, F^{(1)}\right\rangle:=\left\langle\circ, F^{(1)}\right\rangle$ (cf. [29]).

Since, as is easy to see, for each $n \in \mathbb{Z}_{+}$the set $\left\{:\left\langle 0^{\otimes n}, f^{(n)}\right\rangle: \mid f^{(n)} \in \mathcal{D}_{\mathbb{C}}^{\widehat{\otimes} n}\right\}$ is dense in $\mathbf{P}_{n}$, the next statement from (4) follows. 
Theorem 1 (Lytvynov's generalization of CRP, cf. [29]). A random variable $F \in\left(L^{2}\right)$ if and only if there exists a unique sequence of kernels $F^{(n)} \in \mathcal{H}_{\text {ext }}^{(n)}, n \in \mathbb{Z}_{+}$, such that

$$
F=\sum_{n=0}^{\infty}:\left\langle 0^{\otimes n}, F^{(n)}\right\rangle:
$$

(the series converges in $\left(L^{2}\right)$ ) and

$$
\|F\|_{\left(L^{2}\right)}^{2}=\int_{\mathcal{D}^{\prime}}|F(\omega)|^{2} \mu(d \omega)=\mathbb{E}|F|^{2}=\sum_{n=0}^{\infty} n !\left|F^{(n)}\right|_{\text {ext }}^{2}<\infty .
$$

Remark 2. In this paper we do not use directly an explicit formula for the scalar products $(\cdot, \cdot)_{\text {ext }}$, and therefore we prefer not to write it down. But for the interested reader we note that such a formula is calculated in [29]; in another record form (more convenient for some calculations) it is given in, e.g., $[9,11,13]$. Also we note that for each $n \in \mathbb{N}$ the space $\mathcal{H}_{\text {ext }}^{(n)}$ is the symmetric subspace of the space of (classes of) complex-valued functions on $\mathbb{R}_{+}^{n}$, square integrable with respect to a certain Radon measure.

Denote $\mathcal{H}:=L^{2}\left(\mathbb{R}_{+}\right)$, then $\mathcal{H}_{\mathbb{C}}=L^{2}\left(\mathbb{R}_{+}\right)_{\mathrm{C}}$ (in what follows, this notation will be used very often). It follows from the explicit formula for $(\cdot, \cdot)_{\text {ext }}$ that $\mathcal{H}_{\text {ext }}^{(1)}=\mathcal{H}_{\mathbb{C}}$, and for $n \in \mathbb{N} \backslash\{1\}$ one can identify $\mathcal{H}_{\mathbb{C}}^{\widehat{\otimes} n}$ with the proper subspace of $\mathcal{H}_{\text {ext }}^{(n)}$ that consists of "vanishing on diagonals" elements (roughly speaking, such that $F^{(n)}\left(u_{1}, \ldots, u_{n}\right)=0$ if there exist $k, j \in\{1, \ldots, n\}: k \neq j$, but $u_{k}=u_{j}$ ). In this sense the space $\mathcal{H}_{\text {ext }}^{(n)}$ is an extension of $\mathcal{H}_{\mathbb{C}}^{\widehat{\otimes} n}$, this explains why we use the subscript "ext" in our designations.

\subsection{A nonregular rigging of $\left(L^{2}\right)$}

Let $T$ be the set of indexes $\tau=\left(\tau_{1}, \tau_{2}\right)$, where $\tau_{1} \in \mathbb{N}, \tau_{2}$ is an infinite differentiable function on $\mathbb{R}_{+}$such that for all $u \in \mathbb{R}_{+} \tau_{2}(u) \geq 1$. Denote by $\mathcal{H}_{\tau}$ the real Sobolev space on $\mathbb{R}_{+}$of order $\tau_{1}$ weighted by the function $\tau_{2}$, i.e., $\mathcal{H}_{\tau}$ is the completion of $\mathcal{D}$ with respect to the norm generated by the scalar product

$$
(\varphi, \psi)_{\mathcal{H}_{\tau}}=\int_{\mathbb{R}_{+}}\left(\varphi(u) \psi(u)+\sum_{k=1}^{\tau_{1}} \varphi^{[k]}(u) \psi^{[k]}(u)\right) \tau_{2}(u) d u,
$$

here $\varphi^{[k]}$ and $\psi^{[k]}$ are derivatives of order $k$ of functions $\varphi$ and $\psi$ respectively. It is well known (e.g., [3]) that $\mathcal{D}=\underset{\tau \in T}{\operatorname{pr} \lim } \mathcal{H}_{\tau}$ (moreover, one can show that for any $n \in \mathbb{N}^{\widehat{\otimes} n}=\underset{\tau \in T}{\operatorname{pr} \lim } \mathcal{H}_{\tau}^{\widehat{\otimes} n}$ ), and for each $\tau \in T \mathcal{H}_{\tau}$ is densely and continuously embedded into $\mathcal{H} \equiv L^{2}\left(\mathbb{R}_{+}\right)$. Therefore one can consider a chain

$$
\mathcal{D}^{\prime} \supset \mathcal{H}_{-\tau} \supset \mathcal{H} \supset \mathcal{H}_{\tau} \supset \mathcal{D},
$$

where $\mathcal{H}_{-\tau}, \tau \in T$, are the spaces dual of $\mathcal{H}_{\tau}$ with respect to $\mathcal{H}$. Note that by the Schwartz theorem [3] $\mathcal{D}^{\prime}=$ ind $\lim _{\tau \in T} \mathcal{H}_{-\tau}$ (it is convenient for us to consider $\mathcal{D}^{\prime}$ as a topological space with the inductive limit topology). By analogy with [20] one can easily show that the measure $\mu$ of a Lévy white noise is concentrated on $\mathcal{H}_{-\tau}$ with some $\widetilde{\tau} \in T$, i.e., $\mu\left(\mathcal{H}_{-}\right)=1$. Excepting from $T$ the indexes $\tau$ such that $\mu$ is not concentrated on $\mathcal{H}_{-\tau}$, we will assume, in what follows, that for each $\tau \in T \mu\left(\mathcal{H}_{-\tau}\right)=1$. 
Denote the norms in $\mathcal{H}_{\tau, \mathrm{C}}$ and its symmetric tensor powers by $|\cdot|_{\tau}$, i.e., for $f^{(n)} \in \mathcal{H}_{\tau, \mathrm{C}^{\prime}}^{\widehat{\otimes} n}$ $n \in \mathbb{Z}_{+},\left|f^{(n)}\right|_{\tau}=\sqrt{\left(f^{(n)}, \overline{f^{(n)}}\right)_{\mathcal{H}_{\tau, \mathrm{C}}{ }^{\otimes} n}}$ (note that $\mathcal{H}_{\tau, \mathrm{C}}^{\widehat{\otimes} 0}:=\mathbb{C}$ and $\left.\left|f^{(0)}\right|_{\tau}=\left|f^{(0)}\right|\right)$.

It follows from results of [19] that one can modify $T$ again (it is necessary to remove from $T$ some "bad" indexes) in order to obtain the following statement.

Proposition 1. For each $\tau \in T$ and each $n \in \mathbb{Z}_{+}$the space $\mathcal{H}_{\tau, \mathrm{C}}^{\widehat{\otimes} n}$ is densely and continuously embedded into the space $\mathcal{H}_{\text {ext }}^{(n)}$, and there exists $c(\tau)>0$ such that for all $f^{(n)} \in \mathcal{H}_{\tau, \mathrm{C}}^{\widehat{\otimes} n}\left|f^{(n)}\right|_{\text {ext }}^{2} \leq$ $n ! c(\tau)^{n}\left|f^{(n)}\right|_{\tau}^{2}$.

Accept on default $q \in \mathbb{Z}_{+}$and $\tau \in T$. Denote $\mathcal{P}_{W}:=\left\{f=\sum_{n=0}^{N_{f}}:\left\langle 0^{\otimes n}, f^{(n)}\right\rangle:, f^{(n)} \in\right.$ $\left.\mathcal{D}_{\mathrm{C}}^{\widehat{\otimes} n}, N_{f} \in \mathbb{Z}_{+}\right\} \subset\left(L^{2}\right)$. Define real (bilinear) scalar products $(\cdot, \cdot)_{\tau, q}$ on $\mathcal{P}_{W}$ by setting for

$$
\begin{gathered}
f=\sum_{n=0}^{N_{f}}:\left\langle 0^{\otimes n}, f^{(n)}\right\rangle:, g=\sum_{n=0}^{N_{g}}:\left\langle 0^{\otimes n}, g^{(n)}\right\rangle: \in \mathcal{P}_{W} \\
(f, g)_{\tau, q}:=\sum_{n=0}^{\min \left(N_{f}, N_{g}\right)}(n !)^{2} 2^{q n}\left(f^{(n)}, g^{(n)}\right)_{\mathcal{H}_{\tau, \mathrm{C}}^{\otimes n}} .
\end{gathered}
$$

The well-posedness of this definition is proved in [22].

Let $\|\cdot\|_{\tau, q}$ be the norms corresponding to scalar products (7), i.e., $\|\cdot\|_{\tau, q}:=\sqrt{\left(\cdot,^{-}\right)_{\tau, q}}$. De-

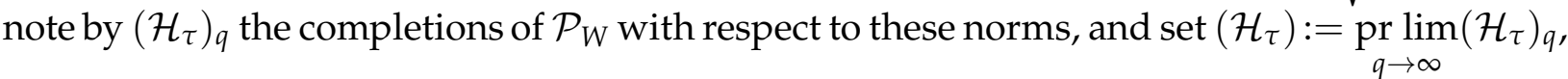
$(\mathcal{D}):=\underset{\tau \in T, q \rightarrow \infty}{\operatorname{pr} \lim }\left(\mathcal{H}_{\tau}\right)_{q}$. As is easy to see, $f \in\left(\mathcal{H}_{\tau}\right)_{q}$ if and only if $f$ can be uniquely presented in the form

$$
f=\sum_{n=0}^{\infty}:\left\langle 0^{\otimes n}, f^{(n)}\right\rangle:, f^{(n)} \in \mathcal{H}_{\tau, \mathrm{C}}^{\widehat{\otimes} n}
$$

(the series converges in $\left(\mathcal{H}_{\tau}\right)_{q}$ ), with

$$
\|f\|_{\tau, q}^{2}:=\|f\|_{\left(\mathcal{H}_{\tau}\right)_{q}}^{2}=\sum_{n=0}^{\infty}(n !)^{2} 2^{q n}\left|f^{(n)}\right|_{\tau}^{2}<\infty
$$

(since for each $n \in \mathbb{Z}_{+} \mathcal{H}_{\tau, \mathrm{C}}^{\widehat{\otimes} n} \subseteq \mathcal{H}_{\text {ext }}^{(n)}$, for $f^{(n)} \in \mathcal{H}_{\tau, \mathrm{C}}^{\widehat{\otimes} n}:\left\langle 0^{\otimes n}, f^{(n)}\right\rangle$ : is a well defined Wick monomial, see Subsection 1.2). Further, $f \in\left(\mathcal{H}_{\tau}\right)(f \in(\mathcal{D}))$ if and only if $f$ can be uniquely presented in form (8) and norm (9) is finite for each $q \in \mathbb{Z}_{+}$(for each $\tau \in T$ and each $q \in \mathbb{Z}_{+}$).

Proposition $2([19,22])$. For each $\tau \in T$ there exists $q_{0}(\tau) \in \mathbb{Z}_{+}$such that for each $q \in \mathbb{N}_{q_{0}(\tau)}:=$ $\left\{q_{0}(\tau), q_{0}(\tau)+1, \ldots\right\}$ the space $\left(\mathcal{H}_{\tau}\right)_{q}$ is densely and continuously embedded into $\left(L^{2}\right)$.

In view of this proposition one can consider a chain

$$
\left(\mathcal{D}^{\prime}\right) \supset\left(\mathcal{H}_{-\tau}\right) \supset\left(\mathcal{H}_{-\tau}\right)_{-q} \supset\left(L^{2}\right) \supset\left(\mathcal{H}_{\tau}\right)_{q} \supset\left(\mathcal{H}_{\tau}\right) \supset(\mathcal{D}), \tau \in T, q \in \mathbb{N}_{q_{0}(\tau)} \text {, }
$$

where $\left(\mathcal{H}_{-\tau}\right)_{-q},\left(\mathcal{H}_{-\tau}\right)=\operatorname{ind}_{q^{\prime} \rightarrow \infty} \lim \left(\mathcal{H}_{-\tau}\right)_{-q^{\prime}}$ and $\left(\mathcal{D}^{\prime}\right)=\operatorname{ind}_{\tilde{\tau} \in T, q^{\prime} \rightarrow \infty}\left(\mathcal{H}_{-\tau}\right)_{-q^{\prime}}$ are the spaces dual of $\left(\mathcal{H}_{\tau}\right)_{q},\left(\mathcal{H}_{\tau}\right)$ and $(\mathcal{D})$ with respect to $\left(L^{2}\right)$. 
Definition 2. Chain (10) is called a nonregular rigging of the space $\left(L^{2}\right)$. The positive spaces of this rigging $\left(\mathcal{H}_{\tau}\right)_{q},\left(\mathcal{H}_{\tau}\right)$ and $(\mathcal{D})$ are called (Kondratiev-type) spaces of nonregular test functions. The negative spaces of this rigging $\left(\mathcal{H}_{-\tau}\right)_{-q},\left(\mathcal{H}_{-\tau}\right)$ and $\left(\mathcal{D}^{\prime}\right)$ are called (Kondratievtype) spaces of nonregular generalized functions.

Finally, we describe natural orthogonal bases in the spaces $\left(\mathcal{H}_{-\tau}\right)_{-q}$. Let us consider chains

$$
\mathcal{D}_{\mathrm{C}}^{\prime(m)} \supset \mathcal{H}_{-\tau, \mathrm{C}}^{(m)} \supset \mathcal{H}_{\text {ext }}^{(m)} \supset \mathcal{H}_{\tau, \mathrm{C}}^{\widehat{\otimes} m} \supset \mathcal{D}_{\mathrm{C}}^{\widehat{\otimes} m},
$$

$m \in \mathbb{N}$, where $\mathcal{H}_{-\tau, \mathrm{C}}^{(m)}$ and $\mathcal{D}_{\mathrm{C}}^{\prime(m)}=\operatorname{ind}_{\widetilde{\tau} \in T} \lim \mathcal{H}_{-\widetilde{\tau}, \mathrm{C}}^{(m)}$ are the spaces dual of $\mathcal{H}_{\tau, \mathbb{C}}^{\widehat{\otimes} m}$ and $\mathcal{D}_{\mathrm{C}}^{\widehat{\otimes} m}$ with respect to $\mathcal{H}_{\text {ext }}^{(m)}$. Set $\mathcal{D}_{\mathrm{C}}^{\widehat{\otimes} 0}=\mathcal{H}_{\tau, \mathrm{C}}^{\widehat{\otimes} 0}=\mathcal{H}_{\text {ext }}^{(0)}=\mathcal{H}_{-\tau, \mathrm{C}}^{(0)}=\mathcal{D}_{\mathrm{C}}^{\prime(0)}:=\mathbb{C}$. In what follows, we denote by $\langle\cdot, \cdot\rangle_{\text {ext }}$ the real (bilinear) dual pairings between elements of negative and positive spaces from chains (11), these pairings are generated by the scalar products in $\mathcal{H}_{\text {ext }}^{(m)}$.

The next statement follows from the definition of the spaces $\left(\mathcal{H}_{-\tau}\right)_{-q}$ and the general duality theory (cf. $[19,20])$.

Proposition 3. There exists a system of generalized functions

$$
\left\{:\left\langle 0^{\otimes m}, F_{\text {ext }}^{(m)}\right\rangle: \in\left(\mathcal{H}_{-\tau}\right)_{-q} \mid F_{\text {ext }}^{(m)} \in \mathcal{H}_{-\tau, \mathrm{C}^{\prime}}^{(m)}, m \in \mathbb{Z}_{+}\right\}
$$

such that

1) for $F_{\text {ext }}^{(m)} \in \mathcal{H}_{\text {ext }}^{(m)} \subset \mathcal{H}_{-\tau, \mathbb{C}}^{(m)}:\left\langle 0^{\otimes m}, F_{\text {ext }}^{(m)}\right\rangle$ : is a Wick monomial that is defined in Subsection 1.2;

2) any generalized function $F \in\left(\mathcal{H}_{-\tau}\right)_{-q}$ can be uniquely presented as a series

$$
F=\sum_{m=0}^{\infty}:\left\langle 0^{\otimes m}, F_{\text {ext }}^{(m)}\right\rangle:, F_{\text {ext }}^{(m)} \in \mathcal{H}_{-\tau, \mathrm{C}^{\prime}}^{(m)}
$$

that converges in $\left(\mathcal{H}_{-\tau}\right)_{-q}$, i.e.,

$$
\|F\|_{-\tau,-q}^{2}:=\|F\|_{\left(\mathcal{H}_{-\tau}\right)_{-q}}^{2}=\sum_{m=0}^{\infty} 2^{-q m}\left|F_{\text {ext }}^{(m)}\right|_{\mathcal{H}_{-\tau, \mathrm{C}}^{(m)}}^{2}<\infty
$$

and, vice versa, any series (12) with finite norm (13) is a generalized function from $\left(\mathcal{H}_{-\tau}\right)_{-q}$ (i.e., such a series converges in $\left.\left(\mathcal{H}_{-\tau}\right)_{-q}\right)$;

3) the dual pairing between $F \in\left(\mathcal{H}_{-\tau}\right)_{-q}$ and $f \in\left(\mathcal{H}_{\tau}\right)_{q}$ that is generated by the scalar product in $\left(L^{2}\right)$, has the form

$$
\langle\langle F, f\rangle\rangle_{\left(L^{2}\right)}=\sum_{m=0}^{\infty} m !\left\langle F_{\text {ext }}^{(m)}, f^{(m)}\right\rangle_{\text {ext }}
$$

where $F_{\text {ext }}^{(m)} \in \mathcal{H}_{-\tau, \mathrm{C}}^{(m)}$ and $f^{(m)} \in \mathcal{H}_{\tau, \mathrm{C}}^{\widehat{\otimes} m}$ are the kernels from decompositions (12) and (8) for $F$ and $f$ respectively.

It is clear that $F \in\left(\mathcal{H}_{-\tau}\right)\left(F \in\left(\mathcal{D}^{\prime}\right)\right)$ if and only if $F$ can be uniquely presented in form (12) and norm (13) is finite for some $q \in \mathbb{N}_{q_{0}(\tau)}$ (for some $\tau \in T$ and some $q \in \mathbb{N}_{q_{0}(\tau)}$ ). 


\subsection{An extended stochastic integral on spaces of nonregular generalized functions}

Decomposition (6) for elements of $\left(L^{2}\right)$ defines an isometric isomorphism (a generalized Wiener-Itô-Sigal isomorphism)

$$
\mathbf{I}:\left(L^{2}\right) \rightarrow \bigoplus_{n=0}^{\infty} n ! \mathcal{H}_{\text {ext }}^{(n)},
$$

where $\bigoplus_{n=0}^{\infty} n ! \mathcal{H}_{e x t}^{(n)}$ is a weighted extended symmetric Fock space: for $F \in\left(L^{2}\right)$ of form (6) $\mathbf{I} F=\left(F^{(0)}, F^{(1)}, \ldots\right) \in \bigoplus_{n=0}^{\infty} n ! \mathcal{H}_{\text {ext }}^{(n)}$. Denote by $\mathbf{1}: \mathcal{H}_{\mathbb{C}} \rightarrow \mathcal{H}_{\mathbb{C}}$ the identity operator. The operator $\mathbf{I} \otimes \mathbf{1}:\left(L^{2}\right) \otimes \mathcal{H}_{\mathbb{C}} \rightarrow\left(\bigoplus_{n=0}^{\infty} n ! \mathcal{H}_{\text {ext }}^{(n)}\right) \otimes \mathcal{H}_{\mathbb{C}} \cong \underset{n=0}{\infty} n !\left(\mathcal{H}_{\text {ext }}^{(n)} \otimes \mathcal{H}_{\mathbb{C}}\right)$ is, obviously, an isometric isomorphism between the spaces $\left(L^{2}\right) \otimes \mathcal{H}_{\mathbb{C}}$ and $\underset{n=0}{\infty} n !\left(\mathcal{H}_{\text {ext }}^{(n)} \otimes \mathcal{H}_{\mathbb{C}}\right)$. It is clear that for arbitrary $m \in \mathbb{Z}_{+}$and $F^{(m)} \in \mathcal{H}_{\text {ext }}^{(m)} \otimes \mathcal{H}_{\mathbb{C}}$ a vector $(\underbrace{0, \ldots, 0}_{m}, F^{(m)}, 0, \ldots)$ belongs to the space $\bigoplus_{n=0}^{\infty} n !\left(\mathcal{H}_{\text {ext }}^{(n)} \otimes \mathcal{H}_{\mathbb{C}}\right)$. Set

$$
:\left\langle\diamond^{\otimes m}, F^{(m)}\right\rangle: \stackrel{\text { def }}{=}(\mathbf{I} \otimes \mathbf{1})^{-1}(\underbrace{0, \ldots, 0}_{m}, F^{(m)}, 0, \ldots) \in\left(L^{2}\right) \otimes \mathcal{H}_{\mathbb{C}}
$$

By the construction elements $:\left\langle 0^{\otimes n}, F^{(n)}\right\rangle:, F^{(n)} \in \mathcal{H}_{\text {ext }}^{(n)} \otimes \mathcal{H}_{\mathrm{C}}, n \in \mathbb{Z}_{+}$, form an orthogonal basis in the space $\left(L^{2}\right) \otimes \mathcal{H}_{\mathbb{C}}$ in the sense that $F \in\left(L^{2}\right) \otimes \mathcal{H}_{\mathbb{C}}$ if and only if $F$ can be uniquely presented as $F \equiv F(\cdot)=\sum_{n=0}^{\infty}:\left\langle 0^{\otimes n}, F^{(n)}\right\rangle:$ (the series converges in $\left(L^{2}\right) \otimes \mathcal{H}_{\mathrm{C}}$ ), with $\|F\|_{\left(L^{2}\right) \otimes \mathcal{H}_{\mathrm{C}}}^{2}=\sum_{n=0}^{\infty} n !\left|F{ }^{(n)}\right|_{\mathcal{H}_{\text {ext }}^{(n)} \otimes \mathcal{H}_{\mathrm{C}}}^{2}<\infty$.

Since, obviously, the restrictions of the generalized Wiener-Itô-Sigal isomorphism $\mathbf{I}$ to the spaces $\left(\mathcal{H}_{\tau}\right)_{q}$ are isometric isomorphisms between $\left(\mathcal{H}_{\tau}\right)_{q}$ and weighted symmetric Fock spaces $\bigoplus_{n=0}^{\infty}(n !)^{2} 2^{q n} \mathcal{H}_{\tau, \mathrm{C}}^{\widehat{\otimes} n}$ (cf. [25]), for arbitrary $n \in \mathbb{Z}_{+}$and $f^{(n)} \in \mathcal{H}_{\tau, \mathrm{C}}^{\widehat{\otimes} n} \otimes \mathcal{H}_{\mathrm{C}} \subset \mathcal{H}_{\text {ext }}^{(n)} \otimes \mathcal{H}_{\mathrm{C}}$ we have $:\left\langle 0^{\otimes n}, f^{(n)}\right\rangle: \in\left(\mathcal{H}_{\tau}\right)_{q} \otimes \mathcal{H}_{\mathrm{C}}$. Moreover, elements $:\left\langle 0^{\otimes n}, f^{(n)}\right\rangle:, f^{(n)} \in \mathcal{H}_{\tau, \mathrm{C}}^{\otimes} \otimes \mathcal{H}_{\mathbb{C}}, n \in \mathbb{Z}_{+}$, form orthogonal bases in the spaces $\left(\mathcal{H}_{\tau}\right)_{q} \otimes \mathcal{H}_{\mathbb{C}}: f \in\left(\mathcal{H}_{\tau}\right)_{q} \otimes \mathcal{H}_{\mathbb{C}}$ if and only if $f$ can be uniquely presented as $f \equiv f(\cdot)=\sum_{n=0}^{\infty}:\left\langle 0^{\otimes n}, f^{(n)}\right\rangle:$ (the series converges in $\left(\mathcal{H}_{\tau}\right)_{q} \otimes \mathcal{H}_{\mathbb{C}}$ ), with $\|f\|_{\left(\mathcal{H}_{\tau}\right)_{q} \otimes \mathcal{H}_{\mathrm{C}}}^{2}=\sum_{n=0}^{\infty}(n !)^{2} 2^{q n}\left|f^{(n)}\right|_{\mathcal{H}_{\tau, \mathrm{C}}^{\otimes n} \otimes \mathcal{H}_{\mathrm{C}}}^{2}<\infty$.

Further, as in the case of spaces $\left(\mathcal{H}_{-\tau}\right)_{-q}$, it follows from the general duality theory that there exists a system of orthogonal in each $\left(\mathcal{H}_{-\tau}\right)_{-q} \otimes \mathcal{H}_{\mathbb{C}}$ generalized functions

$$
\left\{:\left\langle 0^{\otimes m}, F_{\text {ext },}^{(m)}\right\rangle: \in\left(\mathcal{H}_{-\tau}\right)_{-q} \otimes \mathcal{H}_{\mathbb{C}} \mid F_{\text {ext, }}^{(m)} \in \mathcal{H}_{-\tau, \mathbb{C}}^{(m)} \otimes \mathcal{H}_{\mathbb{C}}, m \in \mathbb{Z}_{+}\right\}
$$

such that for $F_{\text {ext, }}^{(m)} \in \mathcal{H}_{\text {ext }}^{(m)} \otimes \mathcal{H}_{\mathbb{C}} \subset \mathcal{H}_{-\tau, \mathrm{C}}^{(m)} \otimes \mathcal{H}_{\mathbb{C}}:\left\langle 0^{\otimes m}, F_{\text {ext },}^{(m)}\right\rangle:$ is given by (15); any generalized function $F \in\left(\mathcal{H}_{-\tau}\right)_{-q} \otimes \mathcal{H}_{\mathrm{C}}$ can be uniquely presented as a convergent in $\left(\mathcal{H}_{-\tau}\right)_{-q} \otimes \mathcal{H}_{\mathrm{C}}$ series

$$
F \equiv F(\cdot)=\sum_{m=0}^{\infty}:\left\langle 0^{\otimes m}, F_{\text {ext },}^{(m)}\right\rangle:, F_{\text {ext, }}^{(m)} \in \mathcal{H}_{-\tau, \mathbb{C}}^{(m)} \otimes \mathcal{H}_{\mathbb{C}}
$$

with

$$
\|F\|_{\left(\mathcal{H}_{-\tau}\right)_{-q} \otimes \mathcal{H}_{\mathrm{C}}}^{2}=\sum_{m=0}^{\infty} 2^{-q m}\left|F_{\text {ext, }}^{(m)}\right|_{\mathcal{H}_{-\tau, \mathrm{C}}^{(m)} \otimes \mathcal{H}_{\mathrm{C}}}^{2}<\infty
$$


and, vice versa, any series (17) with finite norm (18) is a generalized function from $\left(\mathcal{H}_{-\tau}\right)_{-q} \otimes$ $\mathcal{H}_{\mathrm{C}}$ (i.e., such a series converges in $\left.\left(\mathcal{H}_{-\tau}\right)_{-q} \otimes \mathcal{H}_{\mathrm{C}}\right)$. So, system (16) is an orthogonal basis in each space $\left(\mathcal{H}_{-\tau}\right)_{-q} \otimes \mathcal{H}_{\mathbb{C}}$. Moreover, it is clear that $F \in\left(\mathcal{H}_{-\tau}\right) \otimes \mathcal{H}_{\mathbb{C}}:=\operatorname{ind} \lim _{q \rightarrow \infty}\left(\mathcal{H}_{-\tau}\right)_{-q} \otimes \mathcal{H}_{\mathbb{C}}$ $\left(F \in\left(\mathcal{D}^{\prime}\right) \otimes \mathcal{H}_{\mathbb{C}}:=\operatorname{ind}_{\tau \in T, q \rightarrow \infty} \lim _{-\tau}\left(\mathcal{H}_{-q}\right)_{-q} \otimes \mathcal{H}_{\mathbb{C}}\right)$ if and only if $F$ can be uniquely presented in form (17) and norm (18) is finite for some $q \in \mathbb{N}_{q_{0}(\tau)}$ (for some $\tau \in T$ and some $q \in \mathbb{N}_{q_{0}(\tau)}$ ).

Now our aim is to describe the construction of an extended stochastic integral with respect to a Lévy process $L$, that is based on decomposition (17). We need a small preparation.

Consider a family of chains

$$
\mathcal{D}_{\mathrm{C}}^{\prime \widehat{\otimes} m} \supset \mathcal{H}_{-\tau, \mathrm{C}}^{\widehat{\otimes} m} \supset \mathcal{H}_{\mathrm{C}}^{\widehat{\otimes} m} \supset \mathcal{H}_{\tau, \mathrm{C}}^{\widehat{\otimes} m} \supset \mathcal{D}_{\mathbb{C}}^{\widehat{\otimes} m}
$$

$m \in \mathbb{N}$ (as is well known (cf. [3]), $\mathcal{H}_{-\tau, \mathrm{C}}^{\widehat{\otimes} m}$ and $\mathcal{D}_{\mathrm{C}}^{\prime \widehat{\otimes} m}=\underset{\tilde{\tau} \in T}{\operatorname{ind} \lim } \mathcal{H}_{-\widetilde{\tau}, \mathrm{C}}^{\widehat{\otimes} m}$ are the spaces dual of $\mathcal{H}_{\tau, \mathrm{C}}^{\widehat{\otimes} m}$ and $\mathcal{D}_{\mathrm{C}}^{\widehat{\otimes} m}$ respectively). Set $\mathcal{D}_{\mathrm{C}}^{\widehat{\otimes} 0}=\mathcal{H}_{\tau, \mathrm{C}}^{\widehat{\otimes} 0}=\mathcal{H}_{\mathrm{C}}^{\widehat{\otimes} 0}=\mathcal{H}_{-\tau, \mathrm{C}}^{\widehat{\otimes} 0}=\mathcal{D}_{\mathrm{C}}^{\prime}{ }^{\widehat{\otimes} 0}:=\mathbb{C}$. Since the spaces of test functions in chains (19) and (11) coincide, there exists a family of natural isomorphisms

$$
U_{m}: \mathcal{D}_{\mathrm{C}}^{\prime(m)} \rightarrow \mathcal{D}_{\mathrm{C}}^{\prime{ }^{\widehat{\otimes}} m}, m \in \mathbb{Z}_{+},
$$

such that for all $F_{\text {ext }}^{(m)} \in \mathcal{D}_{\mathbb{C}}^{\prime(m)}$ and $f^{(m)} \in \mathcal{D}_{\mathbb{C}}^{\widehat{\otimes} m}$

$$
\left\langle F_{\text {ext }}^{(m)}, f^{(m)}\right\rangle_{\text {ext }}=\left\langle U_{m} F_{\text {ext }}^{(m)}, f^{(m)}\right\rangle
$$

It is easy to see that the restrictions of $U_{m}$ to the spaces $\mathcal{H}_{-\tau, \mathrm{C}}^{(m)}$ are isometric isomorphisms between the spaces $\mathcal{H}_{-\tau, \mathrm{C}}^{(m)}$ and $\mathcal{H}_{-\tau, \mathrm{C}}^{\widehat{\otimes} m}$.

Remark 3. Since $\mathcal{H}_{\text {ext }}^{(1)}=\mathcal{H}_{\mathbb{C}}$, in the case $m=1$ chains (19) and (11) coincide. Thus $U_{1}=\mathbf{1}$ is the identity operator on $\mathcal{D}_{\mathrm{C}}^{\prime(1)}=\mathcal{D}_{\mathrm{C}}^{\prime}$. In the case $m=0 U_{0}$ is, obviously, the identity operator on $\mathbb{C}$.

Definition 3. Let $\Delta \in \mathcal{B}\left(\mathbb{R}_{+}\right)$and $F \in\left(\mathcal{H}_{-\tau}\right)_{-q} \otimes \mathcal{H}_{\mathbb{C}}$. We define an extended stochastic integral with respect to a Lévy process $\int_{\Delta} F(u) \widehat{d} L_{u} \in\left(\mathcal{H}_{-\tau}\right)_{-q}$ by setting

$$
\int_{\Delta} F(u) \widehat{d} L_{u}:=\sum_{m=0}^{\infty}:\left\langle 0^{\otimes m+1}, \widehat{F}_{e x t, \Delta}^{(m)}\right\rangle:
$$

where

$$
\widehat{F}_{\text {ext }, \Delta}^{(m)}:=U_{m+1}^{-1}\left\{\operatorname{Pr}\left[\left(U_{m} \otimes \mathbf{1}\right) F_{\text {ext }}^{(m)}, 1_{\Delta}(\cdot)\right]\right\} \in \mathcal{H}_{-\tau, \mathbb{C}}^{(m+1)},
$$

$\operatorname{Pr}$ is the symmetrization operator (more exactly, the orthoprojector acting for each $m \in \mathbb{Z}_{+}$ from $\mathcal{H}_{-\tau, \mathrm{C}}^{\widehat{\otimes} m} \otimes \mathcal{H}_{\mathbf{C}} \subset \mathcal{H}_{-\tau, \mathrm{C}}^{\widehat{\otimes} m} \otimes \mathcal{H}_{-\tau, \mathrm{C}}$ to $\left.\mathcal{H}_{-\tau, \mathrm{C}}^{\widehat{\otimes} m+1}\right), F_{\text {ext, }}^{(m)} \in \mathcal{H}_{-\tau, \mathrm{C}}^{(m)} \otimes \mathcal{H}_{\mathbf{C}}, m \in \mathbb{Z}_{+}$, are the kernels from decomposition (17) for $F$.

Since

$$
\left|\widehat{F}_{\text {ext, }, \Delta}^{(m)}\right|_{\mathcal{H}_{-\tau, \mathrm{C}}^{(m+1)}}=\left|\operatorname{Pr}\left[\left(U_{m} \otimes \mathbf{1}\right) F_{\text {ext },}^{(m)} 1_{\Delta}(\cdot)\right]\right|_{\mathcal{H}_{-\tau, \mathrm{C}}^{\widehat{\otimes} m+1}} \leq\left|\left(U_{m} \otimes \mathbf{1}\right) F_{\text {ext, }}^{(m)}\right|_{\mathcal{H}_{-\tau, \mathrm{C}}^{\widehat{\otimes} m} \otimes \mathcal{H}_{\mathrm{C}}}=\left|F_{\text {ext, }}^{(m)}\right|_{\mathcal{H}_{-\tau, \mathrm{C}}^{(m)} \otimes \mathcal{H}_{\mathbf{C}}}
$$


and therefore by (21), (13) and (18)

$$
\begin{aligned}
\left\|\int_{\Delta} F(u) \widehat{d} L_{u}\right\|_{-\tau,-q}^{2} & =\sum_{m=0}^{\infty} 2^{-q(m+1)}\left|\widehat{F}_{\text {ext, } \Delta}^{(m)}\right|_{\mathcal{H}_{-\tau, \mathrm{C}}^{(m+1)}}^{2} \\
& \leq 2^{-q} \sum_{m=0}^{\infty} 2^{-q m}\left|F_{\text {ext, },}^{(m)}\right|_{\mathcal{H}_{-\tau, \mathrm{C}}^{(m)} \otimes \mathcal{H}_{\mathrm{C}}}^{2}=2^{-q}\|F\|_{\left(\mathcal{H}_{-\tau}\right)_{-q} \otimes \mathcal{H}_{\mathrm{C}^{\prime}}}^{2}
\end{aligned}
$$

this definition is well-posed and, moreover, the extended stochastic integral

$$
\int_{\Delta} \circ(u) \widehat{d} L_{u}:\left(\mathcal{H}_{-\tau}\right)_{-q} \otimes \mathcal{H}_{\mathrm{C}} \rightarrow\left(\mathcal{H}_{-\tau}\right)_{-q}
$$

is a linear continuous operator.

As appears from the above, an extended stochastic integral can be defined by (21), (22) as a linear continuous operator acting from $\left(\mathcal{H}_{-\tau}\right) \otimes \mathcal{H}_{\mathbb{C}}$ to $\left(\mathcal{H}_{-\tau}\right)$, or from $\left(\mathcal{D}^{\prime}\right) \otimes \mathcal{H}_{\mathbb{C}}$ to $\left(\mathcal{D}^{\prime}\right)$. Exactly the integral

$$
\int_{\Delta} \circ(u) \widehat{d} L_{u}:\left(\mathcal{H}_{-\tau}\right) \otimes \mathcal{H}_{\mathrm{C}} \rightarrow\left(\mathcal{H}_{-\tau}\right)
$$

will be the object of our considerations in the forthcoming section.

Remark 4. As easily appears from results of $[19,21]$, stochastic integral (23) and its extension (24) are generalizations of the extended Skorohod stochastic integral on $\left(L^{2}\right) \otimes \mathcal{H}_{\mathbb{C}}$ [21]. The last integral, in turn, is an extension of the Itô stochastic integral.

Also we note that, in contrast to the regular case $[9,12,13,19]$, integrals (23) and (24) cannot be naturally restricted to the spaces of nonregular test functions, see [23] for details.

Remark 5. It follows from the definition of the extended stochastic integral that for each $\Delta \in$ $\mathcal{B}\left(\mathbb{R}_{+}\right)$

$$
\int_{\Delta} \circ(u) \widehat{d L_{u}}=\int_{\mathbb{R}_{+}} \circ(u) 1_{\Delta}(u) \widehat{d} L_{u} .
$$

One can use this representation for an important generalization. Let a function $F: \mathbb{R}_{+} \rightarrow$ $\left(\mathcal{H}_{-\tau}\right)$ be such that $F \notin\left(\mathcal{H}_{-\tau}\right) \otimes \mathcal{H}_{\mathbb{C}}$, but for some $\Theta \in \mathcal{B}\left(\mathbb{R}_{+}\right)$we have $F(\cdot) 1_{\Theta}(\cdot) \in\left(\mathcal{H}_{-\tau}\right) \otimes$ $\mathcal{H}_{\mathbb{C}}$. It is clear that for any measurable $\Delta \subseteq \Theta$ we have now $F(\cdot) 1_{\Delta}(\cdot) \in\left(\mathcal{H}_{-\tau}\right) \otimes \mathcal{H}_{\mathbb{C}}$, therefore one can define $\int_{\Delta} F(u) \widehat{d} L_{u} \in\left(\mathcal{H}_{-\tau}\right)$ by formula (25).

Finally we note that the operator, adjoint to the extended stochastic integral, is called the Hida stochastic derivative. This derivative is closely connected with so-called operators of stochastic differentiation on spaces of nonregular test functions [24]. All the mentioned operators play an important role in the Lévy white noise analysis.

\subsection{Elements of a Wick calculus}

Let $F \in\left(\mathcal{H}_{-\tau}\right)$. We define an $S$-transform $(S F)(\lambda), \lambda \in \mathcal{D}_{\mathbf{C}}$, as a formal series

$$
(S F)(\lambda):=\sum_{m=0}^{\infty}\left\langle F_{\text {ext }}^{(m)}, \lambda^{\otimes m}\right\rangle_{\text {ext }} \equiv F_{\text {ext }}^{(0)}+\sum_{m=1}^{\infty}\left\langle F_{\text {ext }}^{(m)}, \lambda^{\otimes m}\right\rangle_{\text {ext }},
$$

where $F_{\text {ext }}^{(m)} \in \mathcal{H}_{-\tau, \mathrm{C}}^{(m)}$ are the kernels from decomposition (12) for $F$. In particular, $(S F)(0)=$ $F_{\text {ext }}^{(0)}, S 1 \equiv 1$.

Note that each term in series (26) is well-defined, but the series can diverge. However, the last is not an obstruction in order to construct the Wick calculus. 
Definition 4. For $F, G \in\left(\mathcal{H}_{-\tau}\right)$ and a holomorphic at $(S F)(0)$ function $h: \mathbb{C} \rightarrow \mathbb{C}$ we define a Wick product $F \diamond G$ and a Wick version of $h h^{\diamond}(F)$ by setting formally

$$
F \diamond G:=S^{-1}(S F \cdot S G), \quad h^{\diamond}(F):=S^{-1} h(S F) .
$$

It is obvious that the Wick multiplication $\diamond$ is commutative, associative and distributive over a field $\mathbb{C}$.

Note that a function $h$ can be decomposed in a Taylor series

$$
h(u)=\sum_{m=0}^{\infty} h_{m}(u-(S F)(0))^{m} .
$$

Using this decomposition, it is easy to calculate that $h^{\diamond}(F)=\sum_{m=0}^{\infty} h_{m}(F-(S F)(0))^{\diamond m}$, where $F^{\diamond m}:=\underbrace{F \diamond \cdots \diamond F}_{m \text { times }}=S^{-1}\left[(S F)^{m}\right], F^{\diamond 0}:=1$.

"Coordinate formulas" for the Wick product and for the Wick versions of holomorphic functions (i.e., representations of $F \diamond G, F_{1} \diamond \cdots \diamond F_{n}, n \in \mathbb{N}$, and $h^{\diamond}(F)$ via kernels from decompositions (12) for $F, G, F_{1}, \ldots, F_{h}$, and coefficients from decomposition (27) for $h$ ) are given in [22]. Using these formulas, one can prove the following statement.

Theorem 2 ([22]). $\quad$ 1) Let $F_{1}, \ldots, F_{n} \in\left(\mathcal{H}_{-\tau}\right), n \in \mathbb{N}$. Then $F_{1} \diamond \cdots \diamond F_{n} \in\left(\mathcal{H}_{-\tau}\right)$. Moreover, the Wick multiplication is continuous in the sense that

$$
\left\|F_{1} \diamond \cdots \diamond F_{n}\right\|_{-\tau,-q} \leq \sqrt{\max _{m \in \mathbb{Z}_{+}}\left[2^{-m}(m+1)^{n-1}\right]}\left\|F_{1}\right\|_{-\tau,-(q-1)} \cdots\left\|F_{n}\right\|_{-\tau,-(q-1)},
$$

where $q \in \mathbb{N}$ is such that $F_{1}, \ldots, F_{n} \in\left(\mathcal{H}_{-\tau}\right)_{-(q-1)}$.

2) Let $F \in\left(\mathcal{H}_{-\tau}\right)$ and a function $h: \mathbb{C} \rightarrow \mathbb{C}$ be holomorphic at $(S F)(0)$. Then $h^{\diamond}(F) \in$ $\left(\mathcal{H}_{-\tau}\right)$.

Finally, we will write out a "coordinate formula" for $F \diamond G, F, G \in\left(\mathcal{H}_{-\tau}\right)$, which will be necessary in the next section. We need a small preparation: it is necessary to introduce an analog of the symmetric tensor multiplication on the spaces $\mathcal{H}_{-\tau, C^{\prime}}^{(m)}, m \in \mathbb{Z}_{+}$.

$$
\begin{aligned}
& \text { For } F_{\text {ext }}^{(n)} \in \mathcal{H}_{-\tau, \mathrm{C}}^{(n)} \text { and } G_{\text {ext }}^{(m)} \in \mathcal{H}_{-\tau, \mathrm{C}^{\prime}}^{(m)}, n, m \in \mathbb{Z}_{+} \text {, set } \\
& F_{\text {ext }}^{(n)} \diamond G_{\text {ext }}^{(m)}:=U_{n+m}^{-1}\left\{\operatorname{Pr}\left[\left(U_{n} F_{\text {ext }}^{(n)}\right) \otimes\left(U_{m} G_{\text {ext }}^{(m)}\right)\right]\right\} \equiv U_{n+m}^{-1}\left\{\left(U_{n} F_{\text {ext }}^{(n)}\right) \widehat{\otimes}\left(U_{m} G_{\text {ext }}^{(m)}\right)\right\} \in \mathcal{H}_{-\tau, \mathrm{C}}^{(n+m)} .
\end{aligned}
$$

It follows from properties of operators $U_{m}$ (see Subsection 1.4) and of the symmetric tensor multiplication that the multiplication $\diamond$ is commutative, associative and distributive over a field $\mathbb{C}$. One can show [22] that $\left|F_{\text {ext }}^{(n)} \diamond G_{\text {ext }}^{(m)}\right|_{\mathcal{H}_{-\tau, \mathrm{C}}^{(n+m)}} \leq\left|F_{\text {ext }}^{(n)}\right|_{\mathcal{H}_{-\tau, \mathrm{C}}^{(n)}}\left|G_{\text {ext }}^{(m)}\right|_{\mathcal{H}_{-\tau, \mathrm{C}}^{(m)}}$, and for any $\lambda \in \mathcal{D}_{\mathrm{C}}$ $\left\langle F_{\text {ext }}^{(n)}, \lambda^{\otimes n}\right\rangle_{\text {ext }}\left\langle G_{\text {ext }}^{(m)}, \lambda^{\otimes m}\right\rangle_{\text {ext }}=\left\langle F_{\text {ext }}^{(n)} \diamond G_{\text {ext }}^{(m)}, \lambda^{\otimes n+m}\right\rangle_{\text {ext }}$.

Proposition 4 ([22]). For $F, G \in\left(\mathcal{H}_{-\tau}\right)$

$$
F \diamond G=\sum_{m=0}^{\infty}:\left\langle 0^{\otimes m}, \sum_{k=0}^{m} F_{\text {ext }}^{(k)} \diamond G_{e x t}^{(m-k)}\right\rangle:,
$$

where $F_{\text {ext }}^{(k)} \in \mathcal{H}_{-\tau, \mathbb{C}}^{(k)}, G_{\text {ext }}^{(m-k)} \in \mathcal{H}_{-\tau, \mathbb{C}}^{(m-k)}$, are the kernels from decompositions (12) for $F$ and $G$ respectively. In particular, for $F_{\text {ext }}^{(n)} \in \mathcal{H}_{-\tau, \mathrm{C}}^{(n)}$ and $G_{\text {ext }}^{(m)} \in \mathcal{H}_{-\tau, \mathrm{C}}^{(m)}$

$$
:\left\langle 0^{\otimes n}, F_{\text {ext }}^{(n)}\right\rangle: \diamond:\left\langle 0^{\otimes m}, G_{\text {ext }}^{(m)}\right\rangle:=:\left\langle 0^{\otimes n+m}, F_{\text {ext }}^{(n)} \diamond G_{\text {ext }}^{(m)}\right\rangle::
$$


Remark 6. It is relevant to note that the multiplication $\diamond$ is an extension of an analog of the symmetric tensor multiplication on the spaces $\mathcal{H}_{\text {ext }}^{(m)}, m \in \mathbb{Z}_{+}[22,24]$. Using this fact, one can show that the Wick products and the Wick versions of holomorphic functions, introduced on the spaces of regular and nonregular generalized functions (see [11] and [22] respectively), coincide on the intersections of the mentioned spaces. The interested reader can find a detailed information in [22].

\section{MAIN RESULTS AND EXAMPLES}

\subsection{The Wick multiplication under the sign of an integral}

As is known, some properties of an extended stochastic integral differ from habitual properties of the Lebesgue integral. In particular, for $F \in\left(\mathcal{H}_{-\tau}\right)$ and $H^{(1)} \in \mathcal{H}_{\mathrm{C}}$

$$
\int_{\mathbb{R}_{+}}\left(F \otimes H^{(1)}\right)(u) \widehat{d} L_{u} \equiv \int_{\mathbb{R}_{+}} F \cdot H^{(1)}(u) \widehat{d} L_{u} \neq F \cdot \int_{\mathbb{R}_{+}} H^{(1)}(u) \widehat{d} L_{u},
$$

generally speaking, although $F$ does not depend on $u$. Moreover, in general, the pointwise product $F \cdot \int_{\mathbb{R}_{+}} H^{(1)}(u) \widehat{d} L_{u}$ is undefined. Note that these facts are not directly related with pecularities of the Lévy analysis, and hold true even in the classical Gaussian analysis.

But if one uses the Wick multiplication instead of the pointwise multiplication, it becomes possible to take a time-independent multiplier out of the sign of the extended stochastic integral, as in the Lebesgue integration theory (again, this statement holds true in the Gaussian analysis, in the same way as in the Lévy analysis on the spaces of regular generalized functions [12]). In this subsection we'll explain this in detail.

We begin with a preparation. Let $F_{\text {ext }}^{(n)} \in \mathcal{H}_{-\tau, \mathrm{C}}^{(n)}, G_{\text {ext }}^{(m)} \in \mathcal{H}_{-\tau, \mathrm{C}}^{(m)} \otimes \mathcal{H}_{\mathbf{C}}, n, m \in \mathbb{Z}_{+}$. Using the notation of the previous section, define

$$
F_{\text {ext }}^{(n)} \bar{\diamond} G_{\text {ext, }}^{(m)}:=\left(U_{n+m}^{-1} \otimes \mathbf{1}\right)\left\{(\operatorname{Pr} \otimes \mathbf{1})\left[\left(U_{n} F_{\text {ext }}^{(n)}\right) \otimes\left(\left(U_{m} \otimes \mathbf{1}\right) G_{\text {ext, }}^{(m)}\right)\right]\right\} \in \mathcal{H}_{-\tau, \mathrm{C}}^{(n+m)} \otimes \mathcal{H}_{\mathrm{C}} .
$$

Remark 7. Let $n, m \in \mathbb{Z}_{+}, F_{\text {ext }}^{(n)} \in \mathcal{H}_{-\tau, \mathrm{C}}^{(n)}, G_{\text {ext }}^{(m)} \in \mathcal{H}_{-\tau, \mathrm{C}}^{(m)}$ and $H^{(1)} \in \mathcal{H}_{\mathbb{C}}$. By (31) and (28)

$$
F_{\text {ext }}^{(n)} \bar{\diamond}\left(G_{\text {ext }}^{(m)} \otimes H^{(1)}\right)=\left(F_{\text {ext }}^{(n)} \diamond G_{\text {ext }}^{(m)}\right) \otimes H^{(1)}
$$

(cf. [24]).

It is easy to estimate the norm of $F_{e x t}^{(n)} \bar{\diamond} G_{e x t}^{(m)}$, in the space $\mathcal{H}_{-\tau, \mathrm{C}}^{(n+m)} \otimes \mathcal{H}_{\mathbf{C}}$ : since operators $U_{m}: \mathcal{H}_{-\tau, \mathbb{C}}^{(m)} \rightarrow \mathcal{H}_{-\tau, \mathbb{C}}^{\widehat{\otimes} m}, m \in \mathbb{Z}_{+}$, are isometric isomorphisms (see Subsection 1.4), by (31) we obtain

$$
\begin{aligned}
& \left|F_{\text {ext }}^{(n)} \bar{\nabla} G_{\text {ext },}^{(m)}\right|_{\mathcal{H}_{-\tau, \mathrm{C}}^{(n+m)} \otimes \mathcal{H}_{\mathbb{C}}}=\left|(\operatorname{Pr} \otimes \mathbf{1})\left[\left(U_{n} F_{\text {ext }}^{(n)}\right) \otimes\left(\left(U_{m} \otimes \mathbf{1}\right) G_{\text {ext, }}^{(m)}\right)\right]\right|_{\mathcal{H}_{-\tau, \mathrm{C}}^{\widehat{\otimes} n+m} \otimes \mathcal{H}_{\mathbb{C}}} \\
& \leq\left|U_{n} F_{\text {ext }}^{(n)}\right|_{\mathcal{H}_{-\tau, \mathrm{C}}^{\otimes n}}\left|\left(U_{m} \otimes \mathbf{1}\right) G_{\text {ext },}^{(m)}\right|_{\mathcal{H}_{-\tau, \mathrm{C}}^{\otimes m} \otimes \mathcal{H}_{\mathrm{C}}}=\left|F_{\text {ext }}^{(n)}\right|_{\mathcal{H}_{-\tau, \mathrm{C}}^{(n)}}\left|G_{\text {ext, },}^{(m)}\right|_{\mathcal{H}_{-\tau, \mathrm{C}}^{(m)} \otimes \mathcal{H}_{\mathrm{C}}} .
\end{aligned}
$$

Definition 5. Let $F \in\left(\mathcal{H}_{-\tau}\right)$ and $G \in\left(\mathcal{H}_{-\tau}\right) \otimes \mathcal{H}_{\mathbb{C}}$. We define a Wick product $F \bar{\triangleright} G \in\left(\mathcal{H}_{-\tau}\right) \otimes$ $\mathcal{H}_{\mathbb{C}}$, setting

$$
F \bar{\nabla} G \equiv(F \bar{\triangleright} G)(\cdot):=\sum_{m=0}^{\infty}:\left\langle o^{\otimes m}, \sum_{k=0}^{m} F_{\text {ext }}^{(k)} \bar{\triangleright} G_{\text {ext, }}^{(m-k)}\right\rangle:
$$


where $F_{\text {ext }}^{(k)} \in \mathcal{H}_{-\tau, \mathrm{C}}^{(k)}$ and $G_{\text {ext, }}^{(m-k)} \in \mathcal{H}_{-\tau, \mathrm{C}}^{(m-k)} \otimes \mathcal{H}_{\mathrm{C}}$ are the kernels from decompositions (12) and (17) for $F$ and $G$ respectively (cf. (29)).

Using estimate (33) one can prove by analogy with [22] that this definition is well-posed, and the Wick multiplication $\bar{\nabla}$ is continuous in the sense that for any $q \in \mathbb{N}$ such that $F \in$ $\left(\mathcal{H}_{-\tau}\right)_{-(q-1)}$ and $G \in\left(\mathcal{H}_{-\tau}\right)_{-(q-1)} \otimes \mathcal{H}_{\mathbb{C}}$,

$$
\|F \bar{\triangleright} G\|_{\left(\mathcal{H}_{-\tau}\right)_{-q} \otimes \mathcal{H}_{\mathrm{C}}} \leq\|F\|_{\left(\mathcal{H}_{-\tau}\right)_{-(q-1)}}\|G\|_{\left(\mathcal{H}_{-\tau}\right)_{-(q-1)} \otimes \mathcal{H}_{\mathrm{C}}} .
$$

Remark 8. Let $F, G \in\left(\mathcal{H}_{-\tau}\right)$ and $H^{(1)} \in \mathcal{H}_{\mathbb{C}}$. Using (34), (32) and (29), one can easily show that

$$
F \bar{\triangleright}\left(G \otimes H^{(1)}\right)=(F \diamond G) \otimes H^{(1)} .
$$

Theorem 3. Let $\Delta \in \mathcal{B}\left(\mathbb{R}_{+}\right), F \in\left(\mathcal{H}_{-\tau}\right)$ and $G \in\left(\mathcal{H}_{-\tau}\right) \otimes \mathcal{H}_{\mathbb{C}}$. Then

$$
\int_{\Delta} F \bar{\triangleright} G(u) \widehat{d} L_{u} \equiv \int_{\Delta}(F \bar{\diamond} G)(u) \widehat{d L}_{u}=F \diamond \int_{\Delta} G(u) \widehat{d L_{u}} \in\left(\mathcal{H}_{-\tau}\right) .
$$

Remark 9. It is possible to interpret $G$ as a function acting from $\mathbb{R}_{+}$to $\left(\mathcal{H}_{-\tau}\right)$ and, taking into account the construction of the Wick multiplications $\diamond$ and $\bar{\nabla}$, rewrite equality (36) in a classical form $\int_{\Delta} F \diamond G(u) \widehat{d} L_{u}=F \diamond \int_{\Delta} G(u) \widehat{d} L_{u}$.

Proof. It is sufficient to consider the case $\Delta=\mathbb{R}_{+}$only: if $\Delta \neq \mathbb{R}_{+}$, it is necessary to substitute $G(\cdot) 1_{\Delta}(\cdot)$ instead of $G$.

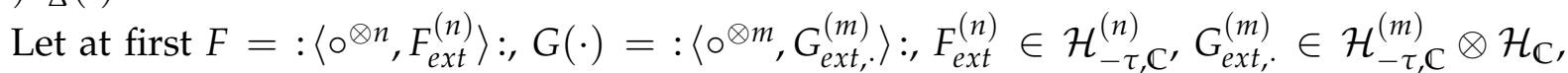
$n, m \in \mathbb{Z}_{+}$. By (34) we have $(F \bar{\diamond} G)(\cdot)=:\left\langle 0^{\otimes n+m}, F_{\text {ext }}^{(n)} \bar{\diamond} G_{\text {ext, }}^{(m)}\right\rangle:$, hence $\int_{\mathbb{R}_{+}}(F \bar{\triangleright} G)(u) \widehat{d} L_{u}=$ $:\left\langle 0^{\otimes n+m+1}, F_{\text {ext }}^{\left.(n) \widehat{\triangleright G_{\text {ext }}^{(m)}}\right)}\right\rangle:$ (see (21), (22)). On the other hand, by (21)

$$
\int_{\mathbb{R}_{+}} G(u) \widehat{d} L_{u}=:\left\langle 0^{\otimes m+1}, \widehat{G}_{e x t, \mathbb{R}_{+}}^{(m)}\right\rangle:,
$$

therefore $F \diamond \int_{\mathbb{R}_{+}} G(u) \widehat{d} L_{u}=:\left\langle 0^{\otimes n+m+1}, F_{\text {ext }}^{(n)} \diamond \widehat{G}_{\text {ext, } \mathbb{R}_{+}}^{(m)}\right\rangle:$ (see (30)). So, we have to prove that

$$
F_{\text {ext }}^{(n)} \widehat{\diamond G_{\text {ext, }}^{(m)}}=F_{+}^{(n)} \diamond \widehat{G}_{\text {ext, } \mathbb{R}_{+}}^{(m)}
$$

in $\mathcal{H}_{-\tau, \mathbb{C}}^{(n+m+1)}$.

Using (22) and (31) we obtain

$$
\begin{aligned}
F_{\text {ext }}^{(n)} \widehat{\nabla G_{\text {ext }, \mathbb{R}_{+}}^{(m)}} & =U_{n+m+1}^{-1}\left\{\operatorname{Pr}\left[\left(U_{n+m} \otimes \mathbf{1}\right)\left(F_{\text {ext }}^{(n)} \bar{\diamond} G_{\text {ext },}^{(m)}\right)\right]\right\} \\
& =U_{n+m+1}^{-1}\left\{\operatorname{Pr}\left[\left(U_{n+m} \otimes \mathbf{1}\right)\left(U_{n+m}^{-1} \otimes \mathbf{1}\right)\left\{(\operatorname{Pr} \otimes \mathbf{1})\left[\left(U_{n} F_{\text {ext }}^{(n)}\right) \otimes\left(\left(U_{m} \otimes \mathbf{1}\right) G_{\text {ext }, .}^{(m)}\right)\right]\right\}\right]\right\} \\
& =U_{n+m+1}^{-1}\left\{\operatorname{Pr}\left[\left(U_{n} F_{\text {ext }}^{(n)}\right) \otimes\left(\left(U_{m} \otimes \mathbf{1}\right) G_{\text {ext }, .}^{(m)}\right)\right]\right\},
\end{aligned}
$$

whereas by (28) and (22)

$$
\begin{aligned}
F_{\text {ext }}^{(n)} \diamond \widehat{G}_{\text {ext }, \mathbb{R}_{+}}^{(m)} & =U_{n+m+1}^{-1}\left\{\operatorname{Pr}\left[\left(U_{n} F_{\text {ext }}^{(n)}\right) \otimes\left(U_{m+1} \widehat{G}_{\text {ext }, \mathbb{R}_{+}}^{(m)}\right)\right]\right\} \\
& =U_{n+m+1}^{-1}\left\{\operatorname{Pr}\left[\left(U_{n} F_{\text {ext }}^{(n)}\right) \otimes\left(U_{m+1} U_{m+1}^{-1}\left\{\operatorname{Pr}\left[\left(U_{m} \otimes \mathbf{1}\right) G_{\text {ext },}^{(m)}\right]\right\}\right)\right]\right\} \\
& =U_{n+m+1}^{-1}\left\{\operatorname{Pr}\left[\left(U_{n} F_{\text {ext }}^{(n)}\right) \otimes\left(\left(U_{m} \otimes \mathbf{1}\right) G_{\text {ext }, .}^{(m)}\right)\right]\right\} .
\end{aligned}
$$

Therefore equality (37) is true, hence in our special case the theorem is proved. In the general case the statement follows from the just obtained result, continuity of the Wick multiplications $\bar{\diamond}$ and $\diamond$, and continuity of operator of stochastic integration (24). 
Now let us obtain an analog of property (36) for a so-called Pettis integral (i.e., for a weak integral) on the spaces of nonregular generalized functions. Denote by $\rho$ the Lebesgue measure on $\mathbb{R}_{+}$and consider $\Delta \in \mathcal{B}\left(\mathbb{R}_{+}\right)$such that $\rho(\Delta)<\infty$. For any $G \in\left(\mathcal{H}_{-\tau}\right) \otimes \mathcal{H}_{\mathbb{C}}$ define the Pettis integral $\int_{\Delta} G(u) d u \in\left(\mathcal{H}_{-\tau}\right)$ as a unique element of $\left(\mathcal{H}_{-\tau}\right)$ such that for each $f \in\left(\mathcal{H}_{\tau}\right)$

$$
\left\langle\left\langle\int_{\Delta} G(u) d u, f\right\rangle\right\rangle_{\left(L^{2}\right)}=\left\langle\left\langle G(\cdot), f \otimes 1_{\Delta}(\cdot)\right\rangle\right\rangle_{\left(L^{2}\right) \otimes \mathcal{H}_{C}} \cdot
$$

Since by the generalized Cauchy-Bunyakovsky inequality for each $q \in \mathbb{N}_{q_{0}(\tau)}$ (see Proposition 2)

$$
\left|\left\langle\left\langle G(\cdot), f \otimes 1_{\Delta}(\cdot)\right\rangle\right\rangle_{\left(L^{2}\right) \otimes \mathcal{H}_{\mathbb{C}}}\right| \leq\|G\|_{\left(\mathcal{H}_{-\tau}\right)_{-q} \otimes \mathcal{H}_{\mathbb{C}}}\|f\|_{\left(\mathcal{H}_{\tau}\right)_{q}} \sqrt{\rho(\Delta)},
$$

this definition is well-posed and the Pettis integral

$$
\int_{\Delta} \circ(u) d u:\left(\mathcal{H}_{-\tau}\right) \otimes \mathcal{H}_{\mathbb{C}} \rightarrow\left(\mathcal{H}_{-\tau}\right)
$$

is a linear continuous operator.

Let $G \in\left(\mathcal{H}_{-\tau}\right), H^{(1)} \in \mathcal{H}_{\mathbb{C}}$. Then

$$
\int_{\Delta}\left(G \otimes H^{(1)}\right)(u) d u \equiv \int_{\Delta} G \cdot H^{(1)}(u) d u=G \cdot \int_{\Delta} H^{(1)}(u) d u .
$$

In fact, for each $f \in\left(\mathcal{H}_{\tau}\right)$ by (38) we have

$$
\begin{aligned}
\left\langle\left\langle\int_{\Delta} G \cdot H^{(1)}(u) d u, f\right\rangle\right\rangle_{\left(L^{2}\right)} & =\left\langle\left\langle G \otimes H^{(1)}(\cdot), f \otimes 1_{\Delta}(\cdot)\right\rangle\right\rangle_{\left(L^{2}\right) \otimes \mathcal{H}_{C}} \\
& =\langle\langle G, f\rangle\rangle_{\left(L^{2}\right)} \int_{\Delta} H^{(1)}(u) d u=\left\langle\left\langle G \cdot \int_{\Delta} H^{(1)}(u) d u, f\right\rangle\right\rangle_{\left(L^{2}\right)} .
\end{aligned}
$$

Let now $F, G \in\left(\mathcal{H}_{-\tau}\right)$ and $H^{(1)} \in \mathcal{H}_{\mathbb{C}}$. Using (35) and (40) we obtain

$$
\begin{aligned}
\int_{\Delta} F \bar{\nabla}\left(\left(G \otimes H^{(1)}\right)(u)\right) d u & \equiv \int_{\Delta}\left(F \bar{\nabla}\left(G \otimes H^{(1)}\right)\right)(u) d u=\int_{\Delta}\left((F \diamond G) \otimes H^{(1)}\right)(u) d u \\
& \equiv \int_{\Delta}(F \diamond G) \cdot H^{(1)}(u) d u=(F \diamond G) \cdot \int_{\Delta} H^{(1)}(u) d u \\
& =F \diamond\left(G \cdot \int_{\Delta} H^{(1)}(u) d u\right)=F \diamond \int_{\Delta} G \cdot H^{(1)}(u) d u \\
& \equiv F \diamond \int_{\Delta}\left(G \otimes H^{(1)}\right)(u) d u .
\end{aligned}
$$

From here, by virtue of continuity of the Wick multiplications $\bar{\nabla}$ and $\diamond$, and continuity of Pettis integral (39), we obtain the following statement (cf. Theorem 3).

Theorem 4. Let $\Delta \in \mathcal{B}\left(\mathbb{R}_{+}\right)$be such that $\rho(\Delta)<\infty, F \in\left(\mathcal{H}_{-\tau}\right)$ and $G \in\left(\mathcal{H}_{-\tau}\right) \otimes \mathcal{H}_{\mathbb{C}}$. Then

$$
\int_{\Delta} F \bar{\nabla} G(u) d u \equiv \int_{\Delta}(F \bar{\triangleright} G)(u) d u=F \diamond \int_{\Delta} G(u) d u \in\left(\mathcal{H}_{-\tau}\right) .
$$


Remark 10. As in the case of the extended stochastic integral, now one can interpret $G$ as a function acting from $\mathbb{R}_{+}$to $\left(\mathcal{H}_{-\tau}\right)$, and rewrite equality (41) in a form $\int_{\Delta} F \diamond G(u) d u=$ $F \diamond \int_{\Delta} G(u) d u$.

\subsection{A representation of the extended stochastic integral via the Pettis integral}

It is well known that in the Gaussian analysis the extended stochastic integral on spaces of generalized functions can be presented as a Pettis integral:

$$
\int_{\Delta} F(u) \widehat{d} W_{u}=\int_{\Delta} F(u) \diamond \dot{W}_{u} d u, \quad \Delta \in \mathcal{B}\left(\mathbb{R}_{+}\right)
$$

Here $W$ is a Wiener process, $\dot{W}$ is a Gaussian white noise. Depending on the spaces under consideration, equality (42) can be formal or can have a rigorous sense. In any case this equality is very useful for applications, in particular, for studying stochastic equations with Wick type nonlinearities.

Remark 11. In a sense, equality (42) is an analog of a formula for replacement of a measure in the Lebesgue integration theory. In particular, $\dot{W}$ is an analog of a Radon-Nikodym derivative.

In the Lévy analysis representation (42) for the extended stochastic integral holds true up to obvious modifications: it is necessary to substitute a Lévy process and a Lévy white noise instead of a Wiener process and a Gaussian white noise respectively. Now on the spaces of regular generalized functions the analog of (42) is a formal equality [12]; in the nonregular case the corresponding analog is a rigorous equality. Let us explain this in detail.

As we saw in Subsection 1.1, a Lévy white noise can be presented in a form $\dot{L}_{u}=\left\langle 0, \delta_{u}\right\rangle$, $u \in \mathbb{R}_{+}$. As is well known (e.g., [3]), for each $u$ the Dirac delta-function $\delta_{u} \in \mathcal{H}_{-\tau}$, therefore $\dot{L}_{u}=\left\langle 0, \delta_{u}\right\rangle=:\left\langle 0, \delta_{u}\right\rangle: \in\left(\mathcal{H}_{-\tau}\right)$. Let $F \in\left(\mathcal{H}_{-\tau}\right) \otimes \mathcal{H}_{\mathbb{C}}$. In this subsection it will be convenient to interpret $F$ as a function acting from $\mathbb{R}_{+}$to $\left(\mathcal{H}_{-\tau}\right)$, so, for $\rho$-almost all $u \in \mathbb{R}_{+}$the Wick product $F(u) \diamond \dot{L}_{u}$ is a well-defined element of $\left(\mathcal{H}_{-\tau}\right)$ (remind that $\rho$ is the Lebesgue measure on $\left.\mathbb{R}_{+}\right)$. For arbitrary $\Delta \in \mathcal{B}\left(\mathbb{R}_{+}\right)$let us define the Pettis integral $\int_{\Delta} F(u) \diamond \dot{L}_{u} d u$ as a unique element of $\left(\mathcal{H}_{-\tau}\right)$ such that for each $f \in\left(\mathcal{H}_{\tau}\right)$

$$
\left\langle\left\langle\int_{\Delta} F(u) \diamond \dot{L}_{u} d u, f\right\rangle\right\rangle_{\left(L^{2}\right)}=\int_{\Delta}\left\langle\left\langle F(u) \diamond \dot{L}_{u}, f\right\rangle\right\rangle_{\left(L^{2}\right)} d u
$$

(cf. (38)). Since it is possible now that $\rho(\Delta)=\infty$, we cannot use the reasoning from Subsection 2.1 and have to prove the correctness of this definition (simultaneously we'll obtain an analog of (42)). It is sufficient to consider the case $\Delta=\mathbb{R}_{+}$: if $\Delta \neq \mathbb{R}_{+}$, one has to substitute $F(\cdot) 1_{\Delta}(\cdot)$ instead of $F$. By (29), (28) and Remark 3 for $\rho$-almost all $u \in \mathbb{R}_{+}$

$$
\begin{aligned}
F(u) \diamond \dot{L}_{u}=F(u) \diamond:\left\langle 0, \delta_{u}\right\rangle: & =\sum_{m=0}^{\infty}:\left\langle 0^{\otimes m+1}, F_{\text {ext, } u}^{(m)} \diamond \delta_{u}\right\rangle: \\
& =\sum_{m=0}^{\infty}:\left\langle 0^{\otimes m+1}, U_{m+1}^{-1}\left\{\operatorname{Pr}\left[\left(U_{m} F_{\text {ext, }}^{(m)}\right) \otimes \delta_{u}\right]\right\}\right\rangle:,
\end{aligned}
$$


therefore by (8), (14) and (20) we obtain

$$
\begin{aligned}
& \int_{\mathbb{R}_{+}}\left\langle\left\langle F(u) \diamond \dot{L}_{u}, f\right\rangle\right\rangle_{\left(L^{2}\right)} d u \\
& =\int_{\mathbb{R}_{+}}\left\langle\left\langle\sum_{m=0}^{\infty}:\left\langle 0^{\otimes m+1}, U_{m+1}^{-1}\left\{\operatorname{Pr}\left[\left(U_{m} F_{\text {ext,u }}^{(m)}\right) \otimes \delta_{u}\right]\right\}\right\rangle:, \sum_{n=0}^{\infty}:\left\langle 0^{\otimes n}, f^{(n)}\right\rangle:\right\rangle\right\rangle_{\left(L^{2}\right)} d u \\
& =\int_{\mathbb{R}_{+}} \sum_{m=0}^{\infty}(m+1) !\left\langle U_{m+1}^{-1}\left\{\operatorname{Pr}\left[\left(U_{m} F_{\text {ext }, u}^{(m)}\right) \otimes \delta_{u}\right]\right\}, f^{(m+1)}\right\rangle_{\text {ext }} d u \\
& =\int_{\mathbb{R}_{+}} \sum_{m=0}^{\infty}(m+1) !\left\langle\left(U_{m} F_{\text {ext, }}^{(m)}\right) \otimes \delta_{u}, f^{(m+1)}\right\rangle d u \\
& =\int_{\mathbb{R}_{+}} \sum_{m=0}^{\infty}(m+1) !\left\langle U_{m} F_{\text {ext }, u}^{(m)}, f^{(m+1)}\left(\cdot \cdot_{1}, \ldots \cdot{ }_{m}, u\right)\right\rangle d u \\
& =\sum_{m=0}^{\infty}(m+1) ! \int_{\mathbb{R}_{+}}\left\langle U_{m} F_{\text {ext }, u}^{(m)}, f^{(m+1)}\left(\cdot \cdot_{1}, \ldots \cdot{ }_{m}, u\right)\right\rangle d u \\
& =\sum_{m=0}^{\infty}(m+1) !\left\langle\left(U_{m} \otimes \mathbf{1}\right) F_{\text {ext, },}^{(m)}, f^{(m+1)}\right\rangle \text {. }
\end{aligned}
$$

Note that the penultimate equality in (44) is valid because, as is easy to verify,

$$
\int_{\mathbb{R}_{+}} \sum_{m=0}^{\infty}(m+1) !\left|\left\langle U_{m} F_{e x t, u}^{(m)} f^{(m+1)}\left(\cdot \cdot_{1}, \ldots \cdot m, u\right)\right\rangle\right| d u<\infty .
$$

On the other hand, by (21), (22), (8), (14) and (20) we obtain

$$
\begin{aligned}
\left\langle\left\langle\int_{\mathbb{R}_{+}} F(u) \widehat{d} L_{u}, f\right\rangle\right\rangle_{\left(L^{2}\right)} & \\
& =\left\langle\left\langle\sum_{m=0}^{\infty}:\left\langle 0^{\otimes m+1}, U_{m+1}^{-1}\left\{\operatorname{Pr}\left[\left(U_{m} \otimes \mathbf{1}\right) F_{\text {ext },}^{(m)}\right]\right\}\right\rangle:, \sum_{n=0}^{\infty}:\left\langle 0^{\otimes n}, f^{(n)}\right\rangle:\right\rangle\right\rangle_{\left(L^{2}\right)} \\
& =\sum_{m=0}^{\infty}(m+1) !\left\langle U_{m+1}^{-1}\left\{\operatorname{Pr}\left[\left(U_{m} \otimes \mathbf{1}\right) F_{\text {ext },}^{(m)}\right]\right\}, f^{(m+1)}\right\rangle_{\text {ext }} \\
& =\sum_{m=0}^{\infty}(m+1) !\left\langle\left(U_{m} \otimes \mathbf{1}\right) F_{\text {ext }, \cdot}^{(m)}, f^{(m+1)}\right\rangle .
\end{aligned}
$$

Comparing (44) and (45) we conclude that for all $f \in\left(\mathcal{H}_{\tau}\right)$ and $\Delta \in \mathcal{B}\left(\mathbb{R}_{+}\right)$

$$
\begin{aligned}
\int_{\Delta}\left\langle\left\langle F(u) \diamond \dot{L}_{u}, f\right\rangle\right\rangle_{\left(L^{2}\right)} d u & =\int_{\mathbb{R}_{+}}\left\langle\left\langle\left(F(u) 1_{\Delta}(u)\right) \diamond \dot{L}_{u}, f\right\rangle\right\rangle_{\left(L^{2}\right)} d u \\
& =\left\langle\left\langle\int_{\mathbb{R}_{+}} F(u) 1_{\Delta}(u) \widehat{d} L_{u}, f\right\rangle\right\rangle_{\left(L^{2}\right)}=\left\langle\left\langle\int_{\Delta} F(u) \widehat{d} L_{u}, f\right\rangle\right\rangle_{\left(L^{2}\right)},
\end{aligned}
$$

therefore by (43) $\int_{\Delta} F(u) \diamond \dot{L}_{u} d u$ is a well-defined element of $\left(\mathcal{H}_{-\tau}\right)$ and, moreover, we have the following statement.

Theorem 5. For arbitrary $\Delta \in \mathcal{B}\left(\mathbb{R}_{+}\right)$and $F \in\left(\mathcal{H}_{-\tau}\right) \otimes \mathcal{H}_{\mathbb{C}}$

$$
\int_{\Delta} F(u) \widehat{d L}_{u}=\int_{\Delta} F(u) \diamond \dot{L}_{u} d u
$$


Remark 12. The extended stochastic integral can be defined by formulas (21), (22) with $\Delta=$ $\mathbb{R}_{+}$as a linear continuous operator acting from $\left(\mathcal{H}_{-\tau}\right) \otimes \mathcal{H}_{-\tau, \mathrm{C}}$ to $\left(\mathcal{H}_{-\tau}\right)$, cf. [23] (now it is impossible to define the integral by a set $\Delta \neq \mathbb{R}_{+}$because a multiplication of an element of $\mathcal{H}_{-\tau, \mathrm{C}}^{(m)} \otimes \mathcal{H}_{-\tau, \mathrm{C}}$ or $\left(\mathcal{H}_{-\tau}\right) \otimes \mathcal{H}_{-\tau, \mathrm{C}}$ by $1_{\Delta}$ is undefined, cf. (22), (25)). It is easy to show that formulas (36) and (46) (with $\Delta=\mathbb{R}_{+}$) hold true for this integral.

Finally we note that all results of Subsections 2.1 and 2.2 hold true for integrands and measurable sets $\Delta$, satisfying the assumptions of Remark 5 .

\subsection{Examples}

In order to illustrate possible applications of our results, we consider some stochastic equations with Wick type nonlinearities.

Example 1. (a linear equation) Let us consider an integral stochastic equation

$$
X_{t}=X_{0}+\int_{0}^{t} F \bar{\nabla} X_{u} d u+\int_{0}^{t} G \bar{\nabla} X_{u} \widehat{d} L_{u}
$$

where $X_{0}, F, G \in\left(\mathcal{H}_{-\tau}\right)$ (we use here the classical notation $\left.\int_{0}^{t} \equiv \int_{[0, t}\right)$. Applying to this equation the S-transform with regard to (41), (36) and (46), and solving the obtained nonstochastic equation, we get

$$
S X_{t}=S X_{0} \cdot \exp \left\{S F t+S G \int_{0}^{t} \lambda(u) d u\right\} .
$$

Applying to this equality the inverse S-transform, we obtain the solution of (47)

$$
X_{t}=X_{0} \diamond \exp ^{\diamond}\left\{F t+G \diamond L_{t}\right\} \in\left(\mathcal{H}_{-\tau}\right)
$$

Example 2. (a Verhulst type equation) Consider an integral stochastic equation

$$
X_{t}=X_{0}+r \int_{0}^{t} X_{u} \diamond\left(N-X_{u}\right) d u+v \int_{0}^{t} X_{u} \diamond\left(N-X_{u}\right) \widehat{d} L_{u}
$$

where $X_{0} \in\left(\mathcal{H}_{-\tau}\right), N, r, v \in \mathbb{R}, N>0, r>0,\left(S X_{0}\right)(0)>0$. Here for $\rho$-almost all $u \in \mathbb{R}_{+}$ we interpret $X_{u}$ as a generalized function, it follows from the solution of (48) (see below) that $X_{u} \in\left(\mathcal{H}_{-\tau}\right)$ and all integrals in (48) are well defined. As in the previous example, applying to (48) the $S$-transform with regard to (46), solving the obtained equation, and applying the inverse $S$-transform, we get the solution

$$
X_{t}=N\left[1+\left(N X_{0}^{\diamond(-1)}-1\right) \diamond \exp ^{\diamond}\left\{-N\left(r t+v L_{t}\right)\right\}\right]^{\diamond(-1)} \in\left(\mathcal{H}_{-\tau}\right)
$$

where $Y^{\diamond(-1)}:=S^{-1}\left(\frac{1}{S Y}\right)$

Remark 13. It is very easy to show that all results of this paper hold true (up to obvious modifications) if we consider the spaces $\left(\mathcal{D}^{\prime}\right)$ and $\left(\mathcal{D}^{\prime}\right) \otimes \mathcal{H}_{\mathbb{C}}$ instead of the spaces $\left(\mathcal{H}_{-\tau}\right)$ and $\left(\mathcal{H}_{-\tau}\right) \otimes \mathcal{H}_{\mathrm{C}}$ respectively. 


\section{REFERENCES}

[1] Benth F.E., Di Nunno G., Lokka A., Oksendal B., Proske F. Explicit representation of the minimal variance portfolio in markets driven by Léry processes. Math. Finance 2003, 13 (1), 55-72.

[2] Berezansky Yu.M., Samoilenko Yu.S. Nuclear spaces of functions of infinitely many variables. Ukr. Math. J. 1973, 25 (6), 599-609.

[3] Berezansky Yu. M., Sheftel Z.G., Us G.F. Functional Analysis, Vol. 2. Birkhäuser Verlag, Basel-Boston-Berlin, 1996. (Russian edition: Vyshcha shkola, Kyiv, 1990.)

[4] Bertoin J. Lévy Processes. Cambridge University Press, Cambridge, 1996.

[5] Bożejko M., Lytvynov E.W., Rodionova I.V. An extended anyon Fock space and noncommutative Meixner-type orthogonal polynomials in infinite dimensions. Russian Math. Surveys 2015, 70 (5), 857-899.

[6] Di Nunno G., Oksendal B., Proske F. Malliavin Calculus for Lévy Processes with Applications to Finance. Universitext. Springer-Verlag, Berlin, 2009.

[7] Di Nunno G., Oksendal B., Proske F. White noise analysis for Léry processes. J. Funct. Anal. 2004, 206 (1), 109 148.

[8] Dyriv M.M., Kachanovsky N.A. On operators of stochastic differentiation on spaces of regular test and generalized functions of Léry white noise analysis. Carpathian Math. Publ. 2014, 6 (2), 212-229. doi:10.15330/cmp.6.2.212229

[9] Dyriv M.M., Kachanovsky N.A. Operators of stochastic differentiation on spaces of regular test and generalized functions in the Léry white noise analysis. Res. Bull. NTUU "KPI" 2014, (4), 36-40.

[10] Dyriv M.M., Kachanovsky N.A. Stochastic integrals with respect to a Levy process and stochastic derivatives on spaces of regular test and generalized functions. Res. Bull. NTUU "KPI" 2013, (4), 27-30.

[11] Frei M.M. Wick calculus on spaces of regular generalized functions of Lévy white noise analysis. Carpathian Math. Publ. 2018, 10 (1), 82-104. doi:10.15330/cmp.10.1.82-104

[12] Frei M.M., Kachanovsky N.A. On the relationship between Wick calculus and stochastic integration in the Léry white noise analysis. Eur. J. Math. 2020, in print, doi:10.1007/s40879-019-00317-8

[13] Frei M.M., Kachanovsky N.A. Some remarks on operators of stochastic differentiation in the Léry white noise analysis. Methods Funct. Anal. Topology 2017, 23 (4), 320-345.

[14] Gelfand I.M., Vilenkin N.Ya. Generalized Functions, Vol. IV: Applications of Harmonic Analysis. Academic Press, New York, London, 1964.

[15] Gihman I.I., Skorohod A.V. Theory of Random Processes, Vol. 2. Nauka, Moscow, 1973.

[16] Hida T. Analysis of Brownian Functionals. In: Carleton mathematical lecture notes, Vol. 13. Carleton University, 1975.

[17] Holden H., Oksendal B., Uboe J., Zhang T.-S. Stochastic Partial Differential Equations-a Modeling, White Noise Functional Approach. Birkhäuser, Boston, 1996.

[18] Itô K. Spectral type of the shift transformation of differential processes with stationary increments. Trans. Am. Math. Soc. 1956, 81, 253-263.

[19] Kachanovsky N.A. Extended stochastic integrals with respect to a Lévy process on spaces of generalized functions. Math. Bull. Shevchenko Sci. Soc. 2013, 10, 169-188.

[20] Kachanovsky N.A. On an extended stochastic integral and the Wick calculus on the connected with the generalized Meixner measure Kondratiev-type spaces. Methods Funct. Anal. Topology 2007, 13 (4), 338-379.

[21] Kachanovsky N.A. On extended stochastic integrals with respect to Léry processes. Carpathian Math. Publ. 2013, 5 (2), 256-278.

[22] Kachanovsky N.A. On Wick calculus on spaces of nonregular generalized functions of Lévy white noise analysis. Carpathian Math. Publ. 2018, 10 (1), 114-132. doi: 10.15330/cmp.10.1.114-132 
[23] Kachanovsky N.A. Operators of stochastic differentiation on spaces of nonregular generalized functions of Lévy white noise analysis. Carpathian Math. Publ. 2016, 8 (1), 83-106. doi:10.15330/cmp.8.1.83-106

[24] Kachanovsky N.A. Operators of stochastic differentiation on spaces of nonregular test functions of Léry white noise analysis. Methods Funct. Anal. Topol. 2015, 21 (4), 336-360.

[25] Kachanovsky N.A., Tesko V.A. Stochastic integral of Hitsuda-Skorokhod type on the extended Fock space. Ukr. Math. J. 2009, 61 (6), 873-907.

[26] Kondratiov Yu.G. Generalized Functions in Problems of Infinite-dimensional Analysis. Ph. D. Thesis. Kyiv, 1978. (in Russian)

[27] Kondratiev Yu.G., Samoilenko Yu.S. The spaces of trial and generalized functions of infinitely many variables. Rep. Math. Phys. 1978, 14 (3), 323-348.

[28] Koshmanenko V.D., Samoilenko Yu.S. Isomorphism of fok space with a space of functions of infinitely many variables. Ukr. Math. J. 1975, 27 (5), 552-555.

[29] Lytvynov E.W. Orthogonal decompositions for Léry processes with an application to the gamma, Pascal, and Meixner processes. Infin. Dimens. Anal. Quantum Probab. Relat. Top. 2003, 6 (1), 73-102.

[30] Meyer P.A. Quantum Probability for Probabilists, In: Lect. Notes in Math., Vol. 1538, Springer-Verlag, Berlin, 1993.

[31] Nualart D., Schoutens W. Chaotic and predictable representations for Léry processes. Stochastic Process. Appl. 2000, 90 (1), 109-122.

[32] Schoutens W. Stochastic Processes and Orthogonal Polynomials. In: Lect. Notes in Statist., Vol. 146. SpringerVerlag, New York, 2000.

[33] Skorohod A.V. Integration in Hilbert Space. Springer-Verlag, Berlin-New York-Heidelberg, 1974.

[34] Solé J.L., Utzet F., Vives J. Chaos expansions and Malliavin calculus for Lévy processes. In: Stoch. Anal. and Appl., Abel Symposium 2. Springer, Berlin, 2007, 595-612.

[35] Surgailis D. On $L^{2}$ and non- $L^{2}$ multiple stochastic integration. In: Lect. Notes in Control and Information Sciences, Vol. 36, Springer-Verlag., Berlin-Heidelberg, 1981, 212-226.

[36] Vershik A.M., Tsilevich N.V. Fock factorizations and decompositions of the $L^{2}$ spaces over general Lévy processes. Russian Math. Surveys 2003, 58 (3), 427-472.

Received 18.03.2019

Качановський М.О., Качановська Т.О. Взаємозв'язок між віківським множенням та інтегруванням на просторах нерегулярних узагальнених функиій в аналізі білого шуму Аеві // Карпатські матем. публ. - 2019. - Т.11, №1. - С. 70-88.

Ми маємо справу з просторами нерегулярних узагальнених функцій в аналізі білого шуму Аеві, які побудовані з використанням литвинівського узагальнення властивості хаотичного розкладу. Наша мета - описати взаємовідносини між віківським множенням та інтегруванням на цих просторах. Точніше, ми показуємо, що, використовуючи віківське множення, можна виносити незалежний від часу множник за знак розширеного стохастичного інтегралу; встановлюємо аналог цього результату для інтегралу Петтіса (слабкого інтегралу); та доводимо теорему про представлення розширеного стохастичного інтегралу через інтеграл Петтіса від віківського добутку вихідної підінтегральної функції на білий шум Аеві. Як приклади застосування наших результатів ми розглядаємо деякі стохастичні рівняння з нелінійностями віківського типу.

Ключові слова і фрази: Процес $\Lambda$ еві, розширений стохастичний інтеграл, інтеграл Петтіса, віківський добуток. 